\title{
The Theory of Vector Valued Fourier Hyperfunctions of Mixed Type. II
}

By

\author{
Shigeaki NAGAMACHI
}

\begin{abstract}
The soft resolution $\left(\mathcal{G}_{(0, p)}^{\prime}, \bar{\partial}\right)$ of the sheaf $\mathcal{O}_{k, l}$ of rapidly decreasing holomorphic functions of $(k, l)$ type is constructed. Using the above resolution, we prove $H_{K}^{n}\left(V, \widetilde{\mathcal{O}}_{k, l}\right)$ $\cong L\left(\mathcal{O}_{k, l}(K), E\right)$.
\end{abstract}

\section{$\S 1$. Introduction}

In the first part of the present paper (S. Nagamachi [4]), which will be referred to as [I], we defined the mixed type Fourier hyperfunctions which take values in a Fréchet space $E$. The purpose of this second part is to prove that the space $H_{K}^{n}\left(V,{ }^{E} \widetilde{\mathcal{O}}_{k, l}\right)$ of $E$-valued Fourier hyperfunctions with support contained in a compact set $K$ is isomorphic to the space $L\left(\mathcal{Q}_{k, l}(K), E\right)$ of continuous linear mappings of $\mathcal{Q}_{k, l}(K)$ into $E$. We proved this theorem in [I] only for $E=\mathbb{C}$ (Theorem 5.13 of [I]).

In Section 2, we study the Fourier transformation for slowly increasing $C^{\text {s }}$ functions and rapidly decreasing distributions. In Section 3, we prepare the theory of cohomology with bounds in an appropriate form.

In Section 4, we construct a soft resolution of the sheaf $\mathcal{O}_{k, l}$ of rapidly decreasing holomorphic functions (Theorem 4.9),

$$
0 \rightarrow \mathcal{Q}_{k, l} \rightarrow \mathcal{G}_{(0,0)}^{\prime} \stackrel{\bar{\partial}}{\rightarrow} \ldots \stackrel{\bar{\partial}}{\rightarrow} \mathcal{G}_{(0, n)}^{\prime} \rightarrow 0
$$

where $\mathcal{Q}_{(0, p)}^{\prime}$ is the sheaf subordinate to the presheal $\left\{\mathcal{G}_{(0, p)}^{\prime}(\Omega)\right\}$ of $(0, p)$-forms whose coefficients are rapidly decreasing distributions in $\Omega$

Communicated by M. Sato, October 27, 1978.

* Department of Mathematics, Faculty of Engineering, Tokushima University, Tokushima 770 , Japan. 
(Definition 4.1). To do this, we use the method similar to that developed in 7.6 of L. Hörmander [1], that is, the duality arguments, using the property of the Fourier transformation (Propositions in $\S 2$ ) and the estimate of the solutions of certain system of linear equations with polynomial coefficients (Proposition 3.5, which is an extension of Theorem 7. 6.11 of L. Hörmander [1]).

Using this method, we construct also the following resolution of $\widetilde{\mathcal{O}}_{k, l}$, on $\boldsymbol{Q}^{k, l}$ :

$$
0 \rightarrow \widetilde{\mathcal{O}}_{k, l} \rightarrow \mathscr{E}_{(0,0)} \stackrel{\bar{\partial}}{\rightarrow} \cdots \stackrel{\bar{\partial}}{\rightarrow} \mathscr{F}_{(0, n)} \rightarrow 0,
$$

which is an extension of Theorem 4.11 of [I], where the resolution has been obtained only on the open subset $\Omega$ of $\boldsymbol{Q}^{k, l}$ satisfying a certain condition.

In Section 5 , we prove $H_{K}^{n}\left(V,{ }^{E} \widetilde{\mathcal{O}}_{k, l}\right) \cong L\left(\mathcal{\sim}_{k, l}(K), E\right)$ (Theorem 5.7) using the Serre-Komatsu duality theorem and properties of tensor products of $E$ with nuclear Fréchet spaces.

We continue to use the same notions and notations as those in [I].

\section{§ 2. Function Spaces}

In this section we study the Fourier transformation for slowly increasing $C^{\infty}$ functions and rapidly decreasing distributions.

Definition 2. 1. Let $K$ be the closure of $\prod_{i=1}^{j}\left(\Gamma_{i} \times B_{i}\right)$ in $Q^{k, l}$, where $\Gamma_{i}$ is the strictly convex closed cone in $\boldsymbol{R}^{2 k_{i}+l_{i}}$ whose vertex is at the origin and $B_{i}$ is the closed ball in $\boldsymbol{R}^{l_{i}}$ whose center is at the origin.

In this section we always denote by $K$ the compact set defined in Definition 2. 1.

We identify $\boldsymbol{C}^{n}$ with $\boldsymbol{R}^{2 n}$ and denote by $\langle x, \eta\rangle$ the inner product in $\boldsymbol{R}^{2 n}$, i.e., $\langle x, \eta\rangle=\sum_{i=1}^{2 n} x_{i} \eta_{i}$.

Definition 2. 2. Let $h_{K, \varepsilon}(\eta)=\sup _{x \in K \cap \boldsymbol{R}^{2 n}}(-\langle x, \eta\rangle+\varepsilon|x|)$. Define $K_{\varepsilon}^{\circ}=\left\{\eta \in \boldsymbol{R}^{2 n} ; h_{K, \varepsilon}(\eta)<\infty\right\} \quad$ and $\quad K^{\circ}=\prod_{i=1}^{j}\left(\Gamma_{i}^{\circ} \times \boldsymbol{R}^{l_{i}}\right), \quad$ where $\quad \Gamma_{i}^{\circ}=$ 
$\left\{\eta \in \boldsymbol{R}^{2 k_{i}+l_{i}} ;\langle x, \eta\rangle>0\right.$ for all $\left.0 \neq x \in \Gamma_{i}\right\}$

Proposition 2.3. $K^{\circ}=\cup_{\varepsilon>0} K_{\varepsilon}^{\circ}$.

Proof. Let $\eta_{i}=\left(\alpha_{i}, \beta_{i}\right) \in \Gamma_{i}^{\circ} \times \mathbb{R}^{l_{i}}$. Then

$$
\begin{aligned}
h_{K, \varepsilon}(\eta) & =\sup _{x \in K \cap \boldsymbol{R}^{2 n}}(-\langle x, \eta\rangle+\varepsilon|x|) \\
& =\sum_{i=1}^{j} \sup _{x_{i} \in \Gamma_{i}}\left(-\left\langle x_{i}, \alpha_{i}\right\rangle+\varepsilon\left|x_{i}\right|\right)+\sum_{i=1}^{j} \sup _{y_{i} \in B_{i}}\left(-\left\langle y_{i}, \beta_{i}\right\rangle+\varepsilon\left|y_{i}\right|\right) \\
& =\sum_{i=1}^{j} h_{\Gamma_{i}, \varepsilon}\left(\alpha_{i}\right)+\sum_{i=1}^{j} h_{B_{i}, \varepsilon}\left(\beta_{i}\right) .
\end{aligned}
$$

$h_{K, \varepsilon}(\eta)<\infty$ implies that $h_{\Gamma_{i}, \varepsilon}\left(\alpha_{i}\right)<\infty$ for all $i$ and this shows that $\left\langle x_{i}, \alpha_{i}\right\rangle>0$ for $0 \neq x_{i} \in \Gamma_{i}$ because if $\left\langle x_{i}, \alpha_{i}\right\rangle \leqq 0$ for some $0 \neq x_{i} \in \Gamma_{i}$, then $-\left\langle t x_{i}, \alpha_{i}\right\rangle+\varepsilon\left|t x_{i}\right|$ tends to infinity as $t \rightarrow \infty$, this is a contradiction. Thus we have $K^{\circ} \supset K_{\varepsilon}^{\circ}$ and $K^{\circ} \supset \cup_{\varepsilon>0} K_{\varepsilon}^{\circ}$. Conversely if $\eta \in K^{\circ}$, then let $\inf _{x_{i} \in \Gamma_{i},\left|x_{i}\right|=1}\left\langle x_{i}, \alpha_{i}\right\rangle=\delta_{i}>0$ and choose $\varepsilon>0$ satisfying $\varepsilon<\delta_{i}$ for all $i$. Then we have $-\left\langle t x_{i}, \alpha_{i}\right\rangle+\varepsilon\left|t x_{i}\right| \leqq 0$ for $x_{i} \in \Gamma_{i},\left|x_{i}\right|=1$ and $t \geqq 0$, consequently $h_{\Gamma_{i}, \varepsilon}\left(\alpha_{i}\right) \leqq 0$ for all $i$. Since $h_{B_{i}, \varepsilon}\left(\beta_{i}\right)<\infty$ for all $i, h_{K, \varepsilon}(\eta)$ $=\sum_{i=1}^{j} h_{\Gamma_{i}, \varepsilon}\left(\alpha_{i}\right)+\sum_{i=1}^{j} h_{B_{i}, \varepsilon}\left(\beta_{i}\right)<\infty$. Thus we have $K^{\circ} \subset \cup_{\varepsilon>0} K_{\varepsilon}^{\circ}$.

Proposition 2. 4. If $\eta=\left(\left(\alpha_{1}, \beta_{1}\right), \cdots,\left(\alpha_{j}, \beta_{j}\right)\right) \in K_{\varepsilon}^{\circ}$, then $\left(\left(t_{1} \alpha_{1}\right.\right.$, $\left.\left.s_{1} \beta_{1}\right), \cdots,\left(t_{j} \alpha_{j}, s_{j} \beta_{j}\right)\right) \in K_{\varepsilon}^{\circ}$ for $t_{i} \geqq 1$ and arbitrary real $s_{i}, 1 \leqq i \leqq j$.

Proof. $\eta \in K_{\varepsilon}^{\circ}$ is equivalent to $h_{\Gamma_{i}, \varepsilon}\left(\alpha_{i}\right)<\infty$ for $1 \leqq i \leqq j$. Since $h_{\Gamma_{i}, \varepsilon}\left(\alpha_{i}\right)=\sup _{x_{i} \in \Gamma_{i},\left|x_{i}\right|=1, s \geq 0}\left(-\left\langle x_{i}, \alpha_{i}\right\rangle+\varepsilon\right) s, \quad h_{\Gamma_{i}, \varepsilon}\left(\alpha_{i}\right)<\infty$ is equivalent to $\inf _{x_{i} \in \Gamma_{i},\left|x_{i}\right|=1}\left\langle x_{i}, \alpha_{i}\right\rangle \geqq \varepsilon . \inf _{x_{i} \in \Gamma_{i},\left|x_{i}\right|=1}\left\langle x_{i}, \alpha_{i}\right\rangle \geqq \varepsilon$ implies $\inf _{x_{i} \in \Gamma_{i}, \mid x_{i} i=1}\left\langle x_{i}, t_{i} \alpha_{i}\right\rangle \geqq \varepsilon$ for $t_{i} \geqq 1$. Thus we have $\left(\left(t_{1} \alpha_{1}, s_{1} \beta_{1}\right), \cdots,\left(t_{j} \alpha_{j}, s_{j} \beta_{j}\right)\right) \in K_{\varepsilon}^{\circ}$.

Corollary 2. 5. Let Int $K_{\varepsilon}^{\circ}$ be the interior of $K_{\varepsilon}^{\circ}$. If $\eta=\left(\left(\alpha_{1}, \beta_{1}\right)\right.$, $\left.\cdots,\left(\alpha_{j}, \beta_{j}\right)\right) \in$ Int $K_{\varepsilon}^{\circ}$, then for $t_{i} \geqq 1$ and arbitrary real $s_{i}, 1 \leqq i \leqq j$, $\eta(t, s)=\left(\left(t_{1} \alpha_{1}, s_{1} \beta_{1}\right), \cdots,\left(t_{j} \alpha_{j}, s_{j} \beta_{j}\right)\right) \in \operatorname{Int} K_{\varepsilon}^{\circ}$.

Proof. If $\eta \in$ Int $K_{\varepsilon}^{\circ}$, then there exists a neighbourhood $T^{\top}$ of zero such that $\eta+V \subset K_{\varepsilon}^{\circ}$. By Proposition 2. 4 we have $\eta(t, s)+V(t, 1) \subset K_{\varepsilon}^{\circ}$, where $V(t, 1)=\{\xi(t, 1) ; \xi \in V\}$ is a neighbourhood of zero. Thus we 
have $\eta(t, s) \in \operatorname{Int} K_{\varepsilon}^{\circ}$.

Proposition 2. 6. Let $0<\delta<\varepsilon$. Then $K_{\varepsilon}^{\circ}$ is strictly contained in $K_{\delta}^{\circ}$, that is, the distance between $K_{\varepsilon}^{\circ}$ and the complement $\left(K_{\delta}^{\circ}\right)^{c}$ of $K_{\delta}^{\circ}$ is positive. Therefore $K_{\varepsilon}^{\circ} \subset$ Int $K_{\dot{\delta}}^{\circ}$.

Proof. Let $\eta \in K_{\varepsilon}^{\circ}$ and $e \in \mathbb{C}^{n}$ with $|e|<\varepsilon-\delta$. Since $\eta \in K_{\varepsilon}^{\circ}$ is equivalent to $\inf _{x_{i} \in \Gamma_{i},\left|x_{i}\right|=1}\left\langle x_{i}, \alpha_{i}\right\rangle \geqq \varepsilon$ for $i=1, \cdots, j$,

$$
\begin{aligned}
\inf _{x_{i} \in \Gamma_{i},\left|x_{i}\right|=1}\left\langle x_{i}, \alpha_{i}+e_{i}\right\rangle & \geqq \inf _{x_{i} \in \Gamma_{i},\left|x_{i}\right|=1}\left\langle x_{i}, \alpha_{i}\right\rangle-\sup _{x_{i} \in \Gamma_{i},\left|x_{i}\right|=1}\left\langle x_{i}, e_{i}\right\rangle \\
& \geqq \varepsilon-(\varepsilon-\delta)=\delta .
\end{aligned}
$$

Thus we have shown that $\eta+e \in K_{\delta}^{\circ}$ for all $\eta \in K_{\varepsilon}^{\circ}$ and $|e|<\varepsilon-\delta$. This shows that $K_{\varepsilon}^{\circ}$ is strictly contained in $K_{8}^{\circ}$.

Proposition 2. 7. Let $f$ be a $C^{N}$ function reith support contained in $K \cap \mathbb{R}^{2 n}$. Suppose there exist positive constants $\delta$ and $C$ such that $\left|D^{\alpha} f(x)\right| \leqq C e^{\delta|x|}$ for all $|\alpha| \leqq N$. Define

$$
\tilde{f}(\zeta)=(2 \pi)^{-n} \int_{\boldsymbol{R}^{2 n}} e^{i\langle x, \zeta\rangle} f(x) d x
$$

Then $\tilde{f}(\zeta)$ is an analytic function defined in $\left\{\zeta \in C^{2 n}, \operatorname{Im} \zeta \in \operatorname{Int} K_{\varepsilon}^{\circ}\right\}$ for any $\varepsilon>\delta$, and satisfies

$$
|\tilde{f}(\zeta)| \leqq C_{\varepsilon}^{\prime} e^{h_{K, \varepsilon}(\operatorname{Im} \zeta)} /(1+|\zeta|)^{N}
$$

for some constant $C_{\varepsilon}^{\prime}>0$ and $\operatorname{Im} \zeta \in K_{\varepsilon}^{\circ}$.

Proof. Let $\operatorname{Im} \zeta \in K_{\varepsilon}^{\circ}$. The inequalities

$$
\begin{aligned}
i \zeta^{\alpha} \tilde{f}(\zeta) i & \leqq(2 \pi)^{-n} \int_{R^{2 n}} e^{(-\langle x, \operatorname{Im} \zeta\rangle+\varepsilon|x|)} e^{-\varepsilon|x|}\left|D^{\alpha} f(x)\right| d x \\
& \leqq C^{\prime \prime} e^{h_{K, \varepsilon}(\operatorname{Im} \zeta)}
\end{aligned}
$$

imply that $\tilde{f}(\zeta)$ is analytic in $\operatorname{Im} \zeta \in \operatorname{Int} K_{\varepsilon}^{\circ}$ and satisfies (2.1) for $\operatorname{Im} \zeta \in K_{\varepsilon}^{\circ}$.

Corollary 2. 8. Let $f \in \mathscr{F}_{c}(K)$ (Definition 2.14 of [I]), then $\tilde{f}(\zeta)$ is an analytic function defined in $\left\{\zeta \in C^{2 n} ; \operatorname{Im} \zeta \in K^{\circ}\right\}$ and satisfies 


$$
|\tilde{f}(\zeta)| \leqq C_{N^{*} \varepsilon} e^{h_{K, \varepsilon}(\operatorname{Im} \zeta)} /(1+|\zeta|)^{N}
$$

in $\operatorname{Im} \zeta \in K_{\varepsilon}^{\circ}$ for any $\varepsilon>0$ and $N>0$, where $C_{N, \varepsilon}$ is a positive number independent of $\zeta$.

Proof. The corollary follows from Propositions 2.3, 2.6 and 2.7.

Proposition 2.9. Let $K$ be the set defined in Defunition 2.1. For any $0<\varepsilon \leqq 1$, there exists an $\eta_{\varepsilon} \in \operatorname{Int} K_{\varepsilon}^{\circ}$ satisfying $\left|\eta_{\varepsilon}\right| \leqq A \varepsilon$ for some positive constant A not depending on $\varepsilon$.

Proof. Let $\eta \in K_{2}^{\circ}$ and $A=|\eta|$. Define $\eta_{\varepsilon}=\varepsilon \eta$ for $0<\varepsilon \leqq 1$, then $\left|\eta_{\varepsilon}\right|=A \varepsilon$ and

$$
h_{K, 2 \varepsilon}\left(\eta_{\varepsilon}\right)=\sup _{x \leqq K \cap \boldsymbol{R}^{2 n}}(-\langle x, \varepsilon \eta\rangle+2 \varepsilon|x|)=\varepsilon h_{K, 2}(\eta)<\infty .
$$

This shows that $\gamma_{\varepsilon} \in K_{2 \varepsilon}^{\circ} \subset \operatorname{Int} K_{\varepsilon}^{\circ}$.

Proposition 2. 10. Let $N \geqq 3 n$, and let $g(\zeta)$ be an analytic function in $\left\{\zeta \in \mathbb{C}^{2 n}: \operatorname{Im} \zeta \in \operatorname{Int} K_{\varepsilon}^{\circ}\right\}$ which satisfies

$$
|g(\zeta)| \leqq C \frac{1}{(1+|\zeta|)^{N}} e^{h_{K . c(\operatorname{Im} \xi)}}
$$

for $\operatorname{Im} \zeta \in K_{\varepsilon}^{\circ}$. If we define

$$
g(x)=(2 \pi)^{-n} \int_{\mathbb{R}^{2 n}+i \eta} e^{i(x, \check{\prime} g} g(\zeta) d \zeta \quad \text { for } \eta \in \operatorname{Int} K_{\varepsilon}^{\circ}
$$

$\hat{g}(x)$ is a $C^{N-3 n}$ function roith support contained in $K \cap \mathbb{R}^{2 n}$, satisfying $\left|D^{a} \hat{g}(x)\right|<M e^{\delta|x|}$ for some constant $M$ and $\delta=A$, where $A$ is the constant appeared in Proposition 2.9.

Proof. The inequalities

$$
\begin{aligned}
|\widehat{g}(x)| & =\left|(2 \pi)^{-n} \int_{\boldsymbol{R}^{2 n}} e^{-i\langle x, \xi\rangle} e^{\langle x, \eta} g(\hat{\xi}+i \eta) d \xi\right| \\
& \leqq C^{\prime} e^{\backslash x, \eta\rangle} \int|g(\hat{\xi}+i \eta)| d \xi \\
& \leqq C e^{\langle l, \eta\rangle} e^{h_{K, \varepsilon}(\eta)}
\end{aligned}
$$


hold for $\eta \in \operatorname{Int} K_{\varepsilon}^{\circ} . \quad x \notin K$ implies $x_{l} \notin \Gamma_{l}$ or $y_{k} \notin B_{k}$ for some $l, k$. Hence there exists $\alpha_{l} \in \Gamma_{l}^{\circ}$ such that $\left\langle x_{l}, \alpha_{l}\right\rangle\left\langle 0\right.$ or $y_{k}$ satisfies $\left\langle y_{k}, s y_{k}\right\rangle$ $>h_{B_{k}, \varepsilon}\left(s y_{k}\right)$ for large $s>0$. Since (2.2) is independent of $\eta \in \operatorname{Int} K_{\varepsilon}^{\circ}$ by the Cauchy-Poincaré theorem, we have, for large $s>0$,

$$
\begin{gathered}
|\widehat{g}(x)| \leqq C \exp \left(\left\langle x_{l}, t \alpha_{l}\right\rangle+\left\langle y_{k},-s y_{k}\right\rangle+h_{B_{k}, \varepsilon}\left(-s y_{k}\right)\right. \\
\quad+\sum_{i \neq l}\left\langle x_{i}, \alpha_{i}\right\rangle+\sum_{i \neq k}\left\langle y_{i}, \beta_{i}\right\rangle+\sum_{i \neq k} h_{B_{i}, \varepsilon}\left(\beta_{i}\right),
\end{gathered}
$$

where we have used the facts that $h_{\Gamma_{i}, \varepsilon}\left(\alpha_{i}\right) \leqq 0$ and $\eta=\left(\left(\alpha_{1}, \beta_{1}\right), \cdots\right.$, $\left.\left(t \alpha_{l}, \beta_{l}\right), \cdots,\left(\alpha_{k}, s y_{k}\right), \cdots,\left(\alpha_{j}, \beta_{j}\right)\right) \in \operatorname{Int} K_{\varepsilon}^{\circ}$ for large $t, s$ (Proposition 2.4). The right hand side of (2.3) vanishes as $t$ or $s$ tends to infinity. Thus we have $g(x)=0$ if $x \notin K$.

Let $|\alpha| \leqq N-3 n$. The inequalities

$$
\begin{aligned}
\left|D^{\alpha} \widehat{g}(x)\right| & =(2 \pi)^{-n}\left|\int_{R^{2 n}} e^{-i\langle x, \xi\rangle} e^{\langle x, \eta\rangle}(-i \xi+\eta)^{\alpha} g(\xi+i \eta) d \xi\right| \\
& \leqq C e^{\langle x, \eta\rangle} e^{h_{K, \varepsilon}(\eta)} \\
& \leqq C e^{|x| \cdot|\eta|} e^{h_{K, \varepsilon}(\eta)}
\end{aligned}
$$

hold for $\eta=\eta_{\varepsilon} \in \operatorname{Int} K_{\varepsilon}^{\circ}$ such that $\left|\eta_{\varepsilon}\right| \leqq \delta=A \varepsilon$ by Proposition 2.9. Hence

$$
\left|D^{\alpha} \widehat{g}(x)\right| \leqq M e^{\delta|\cdot x|}
$$

holds for some constant $M>0$.

Proposition 2. 11. Let $f$ be a $C^{N}$ function satisfying the conditions in Proposition 2.7, then $\widehat{\hat{f}}=f$.

Proof. Let $\eta \in \operatorname{Int} K_{\varepsilon}^{\circ}$, then $e^{-\langle y, \eta\rangle} f(y)$ is rapidly decreasing. Therefore we have

$$
\begin{aligned}
\hat{\tilde{f}}(x) & =(2 \pi)^{-2 n} \int_{\boldsymbol{R}^{2 n}+i \eta} e^{-i\langle x, \zeta\rangle}\left(\int_{\boldsymbol{R}^{2 n}} e^{i\langle y, \zeta\rangle} f(y) d y\right) d \zeta \\
& =(2 \pi)^{-2 n} \int_{\boldsymbol{R}^{2 n}} e^{-i\langle x, \xi\rangle} e^{\langle x, \eta\rangle}\left(\int_{\boldsymbol{R}^{2 n}} e^{i\langle y, \xi\rangle} e^{-\langle y, \eta\rangle} f(y) d y\right) d \xi \\
& =f(x) .
\end{aligned}
$$

Proposition 2. 12. Let $g(\zeta)$ be an analytic function satisfying the condition in Proposition 2.10. Then $\widetilde{\widehat{g}}=g$. 
Proof. Let $\zeta=\xi+i \eta$ and $\eta \in \operatorname{Int} K_{\varepsilon}^{\circ}$, then $g(x+i \eta)$ is integrable with respect to $x$. Therefore we have

$$
\begin{aligned}
\widetilde{\widehat{g}}(\zeta) & =(2 \pi)^{-2 n} \int_{\boldsymbol{R}^{2 n}} e^{i\langle u, \zeta\rangle}\left(\int_{\boldsymbol{R}^{2 n}+i \eta} e^{-i\langle u, z\rangle} g(z) d z\right) d u \\
& =(2 \pi)^{-2 n} \int_{\boldsymbol{R}^{2 n}} e^{-\langle u, \eta\rangle} e^{i\langle u, \xi\rangle}\left(\int_{\boldsymbol{R}^{2 n}} e^{-i\langle u, x\rangle} c^{\langle u, \eta\rangle} g(x+i \eta) d x\right) d u \\
& =g(\xi+i \eta)=g(\zeta) .
\end{aligned}
$$

Proposition 2.13. Let $f \in \mathscr{F}_{c}(K)$, re define

$$
|f|_{N, \varepsilon}^{2}=\int_{\boldsymbol{R}^{2 n-i K_{\varepsilon}^{\circ}}}|\tilde{f}(\zeta)|^{2} e^{-2 h_{K, \varepsilon}(\operatorname{In} \zeta)}\left(1+|\zeta|^{2}\right)^{N} d \lambda
$$

then there exists a seminorm $\|f\|_{M . \delta}=\sup _{x \in \boldsymbol{R}^{2 n},|\alpha| \leq M}\left|e^{-\hat{o}|x|} D^{a} f(x)\right|$ of $\mathscr{E}_{c}(K)$ such that $|f|_{N, \varepsilon} \leqq C\|f\|_{M, o}$.

Proof. The inequalities

$$
\begin{aligned}
& \left|e^{\left.-h_{K, \varepsilon}(\operatorname{Im})^{5}\right)} \zeta^{a} \tilde{f}(\zeta)\right| \\
& =\frac{1}{(2 \pi)^{n}} \mid \int_{\boldsymbol{R}^{2 n}} e^{-h_{\boldsymbol{K}, \varepsilon}(\operatorname{Im} \xi)} e^{i\langle x, \xi\rangle} D^{\alpha} f(x) d x \\
& \leqq \frac{1}{(2 \pi)^{n}}\left|\int_{\boldsymbol{R}^{3 n}} e^{\langle x, \operatorname{Im} \zeta\rangle-\varepsilon ; x \mid} \mathcal{C}^{i\langle x, 5\rangle} D^{\alpha} f(x) d x\right| \\
& \leqq \frac{1}{(2 \pi)^{n}} \int_{\boldsymbol{R}^{2 n}}\left|e^{-\varepsilon_{1} x \mid} D^{\alpha} f(x)\right| d x \\
& \leqq C_{\delta}\|f\|_{N, \delta},
\end{aligned}
$$

for $0<\delta<\varepsilon$ and $\operatorname{Im} \zeta \in K_{\varepsilon}^{\circ}$, show that

$$
e^{-2 h_{K, \varepsilon}(\operatorname{Im} \zeta)}\left(1+|\zeta|^{2}\right)^{N}|\tilde{f}(\zeta)|^{2} \leqq C^{\prime}\|f\|_{N, \delta}^{2} .
$$

Then we have

$$
\begin{aligned}
|f|_{N-3 n, \varepsilon}^{2} & =\int_{\boldsymbol{R}^{2 n+i K_{\varepsilon}^{\circ}}} e^{-2 h_{K, \varepsilon}(\operatorname{Im} \zeta)}\left(1+|\zeta|^{2}\right)^{N-3 n}|\tilde{f}(\zeta)|^{2} d \lambda \\
& \leqq C\|f\|_{N, \delta}^{2} .
\end{aligned}
$$

Thus we have, for $M=N+3 n$ and $\delta=\varepsilon / 2$ 
Proposition 2. 14. Int $K_{\varepsilon}^{\circ}=\bigcup_{\delta>\varepsilon} K_{\delta}^{\circ}$.

Proof. Int $K_{\varepsilon}^{\circ} \supset \bigcup_{\delta>\varepsilon} K_{\tilde{\delta}}^{\circ}$ is clear from Proposition 2.6. Let $\eta \in \operatorname{Int} K_{\varepsilon}^{\circ}$, then there exists a positive number $\gamma$ such that for every $e \in C^{n}$ with $|e| \leqq r, \eta+e \in K_{\varepsilon}^{\circ}$. Thus we have

$$
\begin{aligned}
0 & \geqq \sup _{x_{i} \in \Gamma_{i},\left|x_{i}\right|=1,\left|e_{i}\right| \leq r}\left(-\left\langle x_{i}, \alpha_{i}+e_{i}\right\rangle+\varepsilon\right) \\
& =\sup _{x_{i} \in \Gamma_{i},\left|x_{i}\right|=1}\left(-\left\langle x_{i}, \alpha_{i}\right\rangle+\varepsilon+\gamma\right) .
\end{aligned}
$$

This shows that $\eta \in K_{\varepsilon+r}^{\circ}$ and Int $K_{\varepsilon}^{\circ} \subset \cup_{\delta>\varepsilon} K_{\grave{\delta}}^{\circ}$.

Proposition 2. $\mathbb{1 5}$. Int $K_{\varepsilon}^{\circ}$ is a convex set and $h_{K, \varepsilon}(\eta)$ is a convex function in Int $K_{\varepsilon}^{\circ}$.

Proof. Let $\xi, \eta \in \operatorname{Int} K_{\varepsilon}^{\circ}$, then there exist $\delta>\varepsilon$ such that $\xi, \eta \in K_{\dot{0}}^{\circ}$. For $\lambda, \mu \geqq 0, \lambda+\mu=1$, we have

$$
\begin{aligned}
h_{K, \delta}(\lambda \xi+\mu \eta) & =\sup _{x \in K \cap \boldsymbol{R}^{2 n}}(-\langle x, \lambda \xi+\mu \eta\rangle+\delta|x|) \\
& =\sup _{x \in K \cap \boldsymbol{R}^{2 n}}(-\lambda\langle x, \xi\rangle-\mu\langle x, \eta\rangle+\delta(\lambda+\mu)|x|) \\
& \leqq \lambda h_{K, \delta}(\xi)+\mu h_{K, \delta}(\eta)<\infty .
\end{aligned}
$$

This shows that $\lambda \hat{\xi}+\mu \eta \in K_{\hat{o}}^{\circ} \subset \operatorname{Int} K_{\varepsilon}^{\circ}$. Hence Int $K_{\varepsilon}^{\circ}$ is convex. The equation (2.7) shows that $h_{K, o}(\eta)$ is a convex function defined in $K_{\delta}^{\circ}$, hence $h_{K, \varepsilon}(\eta)$ is convex in Int $K_{\varepsilon}^{\circ}$.

Proposition 2. 16. $h_{K, \varepsilon}(\eta)$ is Lipschitz continuous in $K_{\varepsilon}^{\circ}$, that is,

$$
\left|h_{K, \varepsilon}(\eta)-h_{K, \varepsilon}\left(\eta^{\prime}\right)\right| \leqq C\left|\eta-\eta^{\prime}\right|
$$

for some constant $C$.

Proof. Let $\quad h_{\Gamma_{i}, \varepsilon}\left(\alpha_{i}\right)=\sup _{x_{i} \in \Gamma_{i}}\left(-\left\langle x_{i}, \alpha_{i}\right\rangle+\varepsilon\left|x_{i}\right|\right) \quad$ and $\quad h_{B_{i}, \varepsilon}\left(\beta_{i}\right)$ $=\sup _{y_{i} \in B_{i}}\left(-\left\langle y_{i}, \beta_{i}\right\rangle+\varepsilon\left|y_{i}\right|\right)$. Then

$$
h_{K, \varepsilon}(\eta)=\sum_{i=1}^{j} h_{\Gamma_{1}, \varepsilon}\left(\alpha_{i}\right)+\sum_{i=1}^{j} h_{B_{i}, \varepsilon}\left(\beta_{i}\right)
$$

where $\eta=\left(\left(\alpha_{1}, \beta_{1}\right), \cdots,\left(\alpha_{i}, \beta_{i}\right)\right)$ and $x=\left(\left(x_{1}, y_{1}\right), \cdots,\left(x_{i}, y_{j}\right)\right)$. Let $\left|B_{i}\right|$ 
be the diameter of the ball $B_{i}$. We have

$$
\left|h_{B_{i}, \varepsilon}\left(\beta_{i}\right)-h_{B_{i}, \varepsilon}\left(\beta_{i}^{\prime}\right)\right| \leqq \sup _{x_{i} \in B_{i}}\left|\left\langle x_{i}, \beta_{i}-\beta_{i}^{\prime}\right\rangle\right| \leqq\left|B_{i}\right|\left|\beta_{i}-\beta_{i}^{\prime}\right| .
$$

Since $\eta \in K_{\varepsilon}^{\circ}$ implies that $h_{\Gamma_{i}, \varepsilon}\left(\alpha_{i}\right)=0$ for all $i$, we have

$$
\left|h_{K, \varepsilon}(\eta)-h_{K, \varepsilon}\left(\eta^{\prime}\right)\right| \leqq C\left|\eta-\eta^{\prime}\right|
$$

where $C=\max _{i}\left(\left|B_{i}\right|\right)$.

\section{§3. Cohomology with Bounds}

For the later use, we develope the theory of cohomology with bounds on the pseudoconvex domain $\Omega$ in $\boldsymbol{C}^{n}$, which is an extension of what is developed in 7.6 of L. Hörmander [1], where the case $\Omega=C^{n}$ is treaded.

Here we use the same notation that is used in 7.6 of L. Hörmander [1]. We denote by $U^{(\nu)}$ the covering of $\mathbb{C}^{n}$ which consists of the cubes $U_{g}^{(\nu)}$ with side equal to $2 \cdot 3^{-\nu}$ and center at $g \cdot 3^{-\nu}$, where $g$ runs through the set $I$ of points in $C^{n}$ with integral coordinates. For every $\nu$ and $g$ we can find precisely one $g^{\prime}$ such that $U_{g^{\prime}}^{(y)}$ contains the cube with the same center as $U_{g}^{(\nu+1)}$ but twice the side; we set $\rho_{\nu, \nu+1} g=g^{\prime}$. More generally if $\nu<\iota$, we define

$$
\rho_{\nu, \mu} g=\rho_{\nu, \nu+1} \rho_{\nu+1, \nu+2} \cdots \rho_{\mu-1, \mu} g .
$$

Let $\Omega$ be an open subset of $\boldsymbol{C}^{n}$, then $\mathcal{U}^{(\nu)} \cap \Omega=\left\{U_{g}^{(\nu)} \cap \Omega ; g \in I\right\}$ is an open covering of $\Omega$. We also define

$$
\Omega^{\nu, \mu}=\cup\left\{U_{g}^{(\mu)} ; U_{g^{\prime}}^{(\nu)} \subset \Omega \quad \text { for } g^{\prime}=\rho_{\nu, \mu} g\right\}
$$

and

$$
\Omega_{\varepsilon}=\left\{z \in \Omega ; \operatorname{dist}\left(z, \Omega^{c}\right)>\varepsilon\right\}
$$

where dist $\left(z, \Omega^{c}\right)$ is the distance between the point $\approx$ and the complement $\Omega^{c}$ of $\Omega$. We use the abbreviation $\Omega_{\varepsilon}^{\nu, \mu}$ for $\left(\Omega_{\varepsilon}\right)^{\nu, \mu}$.

Let $P=\left(P_{j, k}\right), j=1, \cdots, p, k=1, \cdots, q$ be the matrix with polynomial entries, and consider the sheaf homomorphism

$$
P: O^{q} \rightarrow \Theta^{p}
$$

defined by the mapping $\left(f_{i}, \cdots, f_{q}\right) \in \mathcal{U}^{q}$ to $\left\{\sum P_{j, k} J_{k}\right\}_{j=1}^{p}$. Let $\mathcal{L}_{P}$ be the 
kernel of the sheaf homomorphism (3.1). It is known that $\mathcal{R}_{P}$ is a coherent analytic sheaf and finitely generated by the germs of $q$-tuples $Q=\left(Q_{1}, \cdots, Q_{q}\right)$ with polynomial components such that

$$
\sum_{k=1}^{q} P_{j, k} Q_{k}=0, \quad j=1, \cdots, p
$$

(See Lemma 7.6. 3 of L. Hörmander [1].)

If $\phi$ is a continuous function, we define $C^{\sigma}\left(\mathcal{U}^{(\nu)} \cap \Omega, \mathcal{R}_{P}, \phi\right)$ as the set of alternating cochains $c=\left\{c_{s}\right\}, s \in I^{\sigma^{\perp 1}}$ where $c_{s} \in \Gamma\left(U^{(\nu)} \cap \Omega, \mathscr{R}_{P}\right)$, and

$$
\|c\|_{\phi}=\sum_{|s|=\sigma+1} \int_{U_{s}^{(\nu)} \cap \Omega}\left|c_{s}\right|^{2} e^{-\phi} d \lambda<\infty .
$$

We define $\rho_{\nu, \mu}^{*}: C^{\sigma}\left(U^{(\nu)} \cap \Omega, R_{P}, \phi\right) \rightarrow C^{\sigma}\left(U^{(\mu)} \cap \Omega, R_{P}, \phi\right)$ by setting $\left(\rho_{\nu, \mu}^{*} c\right)_{s}$ equal to the restriction of $c_{\rho_{y, \mu}\left(s_{0}\right) \ldots \rho_{y, \mu}\left(s_{\sigma}\right)}$ to $U_{s}^{(\mu)}$.

Proposition 3. 1. Let $\phi$ be a plurisubharmonic function in an open set $V$ in $C^{n}$, and $\Omega$ be a pseudoconvex dontain contained in $V$. For every cochain $c \in C^{\sigma}\left(U^{(\nu)} \cap V, \Theta, \phi\right)$ with $\delta c=0$, one can find a cochain $c^{\prime} \in C^{\sigma-1}\left(U^{(\nu+\sigma-1)} \cap \Omega^{\nu, \nu-\sigma-1}, \mathcal{O}, \psi\right)$ so that $\delta c^{\prime}=\rho_{\nu, \nu+\sigma-1}^{*} c$ and

$$
\left\|c^{\prime}\right\|_{\psi} \leqq K\|c\|_{\phi}
$$

Here $K$ is a constant independent of $\phi$ and $c$, and $\psi$ is defined by $\psi(z)=\phi(z)+2 \log \left(1+|z|^{2}\right)$.

We prove this in a way similar to Proposition 7.6.1 of L. Hörmander [1], so that we need the following lemma.

Lemma 3.2. Let $\Omega$ be a pseudoconvex domain and let $\Omega^{\prime}$ be a relatively compact subset of $\Omega$. For every plurisubharmonic function $\phi$ in $\Omega$ and every $f \in L_{(0, q+1)}^{2}(\Omega, \phi)$ with $\bar{\partial} f=0$, one can find $u \in L_{(0, q)}^{2}(\Omega$, loc) with $\bar{\partial} u=f$ and

$$
\int_{\Omega^{\prime}}|u|^{2} e^{-\phi} d \lambda \leqq K \int_{\Omega}|f|^{2} e^{-\phi} d \lambda
$$

where $K$ is independent of $u$ and $\phi$.

Proof. See Lemma 7.6.2 of L. Hörmander [1]. 
Proof of Proposition 3.1. We introduce the space $C^{p}\left(U^{(\nu)} \cap V\right.$, $\left.Z_{q}, \phi\right)$ of all alternating cochains $c=\left\{c_{s}\right\}, s \in I^{p+1}$, where $c_{s} \in L_{(0, q)}^{2}\left(U_{s}^{(\nu)}\right.$ $\left.\cap \nu^{\tau}, \phi\right), \bar{\partial} c_{s}=0$ and

$$
\|c\|_{\phi}^{2}=\sum_{|s|=p+1} \int_{U_{s}^{(y)} \cap V}\left|c_{s}\right|^{2} e^{-\phi} d \lambda<\infty .
$$

We wish to prove that if $\delta c=0(p>0)$, then one can find $c^{\prime} \in$ $C^{p-1}\left(U^{(\nu+p-1)} \cap \Omega^{\nu, \nu+p-1}, \mathscr{Z}_{q}, \psi\right)$ so that $\delta c^{\prime}=\rho_{\nu, \nu+p-1}^{*} c$ and (3.2) hold. For $q=0$, this assertion is precisely Proposition 3.1. We shall prove it assuming, if $p>1$, that it has already been proved for smaller values of $p$ and all $q$.

Choose a non-negative function $\chi \in C_{0}^{\infty}\left(U_{0}^{(\nu)}\right)$ such that $\sum_{g} \chi(\approx-g)$ $=1$. Now set $b_{s}=\sum \chi(z-g) c_{g, s}, s \in I^{p}$, then we have $\delta b=c$ and

$$
\left|b_{s}\right|^{2} \leqq \sum \chi(z-g)\left|c_{g, s}\right|^{2}
$$

hence

$$
\|b\|_{\phi}^{2} \leqq\|c\|_{\phi}^{2} \text {. }
$$

Let $\bar{\partial} b$ be the cochain belonging to $C^{p-1}\left(\mathcal{U}^{(\nu)} \cap V, \mathscr{Z}_{q+1}, \phi\right)$ defined by $(\bar{\partial} b)_{s}=\bar{\partial} b_{s}=\sum \bar{\partial} \chi(z-g) \wedge c_{g, s}$. Then we obtain with a constant $K$

$$
\|\bar{\partial} b\|_{\phi} \leqq K\|c\|_{\phi} .
$$

Now $\delta \bar{\partial} b=\bar{\partial} \delta b=\bar{\partial} c=0$. If $p>1$, we can by the inductive hypothesis find a cochain $b^{\prime} \in C^{p-2}\left(\mathcal{U}^{(\nu+p-2)} \cap \Omega^{\nu, \nu-p-2}, \mathscr{Z}_{q+1}, \psi\right)$ such that $\delta b^{\prime}$ $=\rho_{\nu, \nu+p-2}^{*} \bar{\partial} b$ and for some constant $K_{1}$

$$
\left\|b^{\prime}\right\|_{\psi} \leqq K_{1}\|\bar{\partial} b\|_{\phi} \leqq K K_{1}\|c\|_{\phi} .
$$

Since $\bar{\partial} b_{s}^{\prime}=0$ and $\psi$ is plurisubharmonic, by Lemma 3.2 we can choose $b_{s}^{\prime \prime} \in L_{(0, q)}^{2}\left(U_{s}^{(\nu+p-1)}, \psi\right)$ lor every $s \in I^{p-1}$ satisfying $U_{s^{\prime}}^{(\nu+p-2)} \subset \Omega^{\nu, \nu \cdot p-2}, s^{\prime}$ $=\rho_{\nu+p-2, \nu+p-1} s$ so that $\bar{\partial} b_{s}^{\prime \prime}=b_{s^{\prime}}^{\prime}$ in $U_{s}^{(\nu+p-1)}$ and with a constant $K_{2}$,

$$
\int_{U_{s}^{(\nu+p-1)}}\left|b_{s}^{\prime \prime}\right|^{2} e^{-\psi} d \lambda \leqq K_{2} \int_{U_{s}^{(\nu+p-2)}}\left|b_{s^{\prime}}^{\prime}\right|^{2} e^{-\psi} d \lambda .
$$

Now set

$$
c^{\prime}=\rho_{\nu, \nu+p-1}^{*} b-\delta b^{\prime \prime} .
$$

Then $\delta c^{\prime}=\rho_{\nu, \nu+p-1}^{*} \delta b=\rho_{\nu, y+p-1}^{*} c$, and

$$
\bar{\partial} c^{\prime}=\rho_{\nu, \nu+p-1}^{*} \bar{\partial} b-\delta \bar{\partial} b^{\prime \prime}=\rho_{\nu, \nu+p-1}^{*} \bar{\partial} b-\delta \rho_{\nu+p-2, \nu+p-1}^{*} b^{\prime}
$$




$$
=\rho_{\nu, \nu+p-1}^{*} \bar{\partial} b-\rho_{\nu+p-2, \nu+p-1}^{*} \rho_{\nu, \nu+p-2}^{*} \bar{\partial} b=0 .
$$

Summing up the estimates for $b, b^{\prime}$ and $b^{\prime \prime}$ given above, we obtain $c^{\prime} \in C^{p-1}\left(\mathcal{U}^{(\nu+p-1)} \cap \Omega^{\nu, \nu+p-1}, \mathscr{Z}_{q}, \psi\right)$ and the estimate (3.2).

It remains to consider the case $p=1$. The fact that $\delta \bar{\partial} b=0$ then means that $\bar{\partial} b$ defines uniquely a form $f$ of type $(0, q+1)$ in $V$ with $\bar{\partial} f=0$ and

$$
\int|f|^{2} e^{-\phi} d \lambda \leqq\|\bar{\partial} b\|_{\phi}^{2} \leqq K^{2}\|c\|_{\phi}^{2} .
$$

By Theorem 4.4.2 of L. Hörmander [1], we can find a form $u \in$ $L_{(0, q)}^{2}(\Omega, \psi)$ so that $\bar{\partial} u=f$ and

$$
\int_{\Omega}|u|^{2} e^{-\psi} d \lambda \leqq \int_{\Omega}|f|^{2} e^{-\phi} d \lambda
$$

Setting $c_{s}^{\prime}=b_{s}-u$, we obtain $c^{\prime} \in C^{0}\left(U^{(\nu)} \cap \Omega, \mathscr{Z}_{q}, \psi\right)$ and the estimate (3. 2).

Proposition 3. 3. Let $P$ be a matrix with polynomial entries and $\Omega$ be a neighbourhood of 0 . Then there exists a neighbourhood $\Omega^{\prime}$ of 0 such that for every $u \in \mathcal{O}(\Omega+z)^{q}$ one can find $v \in \mathcal{O}\left(\Omega^{\prime}+z\right)^{q}$ satisfying $P v=P u$, and

$$
\sup _{\Omega^{\prime}+z}|v| \leqq C(1+|z|)^{N} \sup _{\Omega+z}|P u|,
$$

where the constants $C$ and $N$ are independent of $u$ and $z \in \mathbb{C}^{n}$.

Proof. See Proposition 7.6.5 of L. Hörmander [1].

Proposition 3. 4. Let a matrix $P$ and an integer $\nu$ be given. Then there exist integers $\mu$ and $N$ such that, if $\phi$ is plurisubharmonic in a pseudoconvex domain $\Omega$ and for some constant $C>0$

$$
\left|\phi(z)-\phi\left(z^{\prime}\right)\right|<C, \quad\left|z-z^{\prime}\right|<1,
$$

then for every $c \in C^{\sigma}\left(U^{(\nu)} \cap \Omega^{\alpha, \nu}, \mathscr{R}_{P}, \phi\right)$ with $\delta c=0, \sigma>0, \lambda \leqq \nu$, one can find $c^{\prime} \in C^{\sigma-1}\left(U^{(\mu)} \cap \Omega_{(\Sigma-\sigma+1) \varepsilon}^{\lambda, \mu}, \mathcal{R}_{P}, \phi_{N}\right)$ so that $\delta c^{\prime}=\rho_{\nu, \mu}^{*} c$ and for some constant $K$

$$
\left\|c^{\prime}\right\|_{\phi_{N}} \leqq K\|c\|_{\phi}
$$


Here $\phi_{N}(z)=\phi(z)+N \log \left(1+|z|^{2}\right), \tau=2^{2 n}$ and $\varepsilon \geq \sqrt{2 n} 3^{1-\lambda}$.

Proof. We can also prove the proposition in a way similar to the proof of Theorem 7.6.10 of L. Hörmander [1]. We shall prove it by induction for decreasing $\sigma$, noting that it is valid when $\sigma>2^{2 n}$, since there are no non-zero $c \in C^{\sigma}\left(U^{(\nu)} \cap \Omega^{k, \nu}, \mathscr{R}_{P}, \phi\right)$. Thus assume that the theorem has been proved for all $P$ when $\sigma$ is replaced by $\sigma+1$. By Lemma 7.6. 4 of L. Hörmander [1], we have $c_{s}=Q d_{s}$ for $d \in C\left(\mathcal{U}^{(\nu)}\right.$ $\left.\cap \Omega^{\lambda, \nu}, \mathcal{O}^{r}\right)$. By Proposition 3.3 and the condition (3.4), if $/$ is large we can choose $d_{s}^{\prime} \in \mathcal{Q}\left(U_{s}^{(\mu)}\right)^{r}$ so that $Q d_{s}^{\prime}=Q d_{s^{\prime}}=c_{s^{\prime}}$ in $U_{s}^{(\mu)}$ and

$$
\int_{U_{s}^{(n)}}\left|d_{s}^{\prime}\right|^{2}\left(1+|z|^{2}\right)^{-N} e^{-\phi(z)} d \lambda \leqq C \int_{U_{s^{\prime}}^{(y)}}\left|c_{s^{\prime}}\right|^{2} e^{-\phi(z)} d \lambda
$$

for $s^{\prime}=\rho_{\nu, \mu} s$ and $U_{s^{\prime}}^{(\nu)} \subset \Omega^{\lambda, \nu}$. Thus we have $d^{\prime} \in C^{\sigma}\left(U^{(\mu)} \cap \Omega^{\lambda, \mu},()^{r}, \phi_{N}\right)$, $\rho_{\nu, \mu}^{*} c=Q d^{\prime}$ and

$$
\left\|d^{\prime}\right\|_{\phi_{N}} \leqq C_{1}\|c\|_{\phi} \text {. }
$$

Since $\delta c=0$, it follows that $\delta Q d^{\prime}=Q \delta d^{\prime}=0$. Thus $\delta d^{\prime}=d^{\prime \prime} \in C^{\sigma+1}\left(\mathcal{U}^{(p)}\right.$ $\left.\cap \Omega^{2, n}, \mathscr{R}_{Q}, \phi_{N}\right)$, and since $\delta d^{\prime \prime}=0$ and $\phi_{N}$ is plurisubharmonic, it follows by the inductive hypothesis that for suitable $N^{\prime}$ and $\mu^{\prime}>\mu$ we can find $d^{\prime \prime \prime} \in C^{\sigma}\left(\mathcal{U}^{\left(\mu^{\prime}\right)} \cap \Omega_{(\tau-\sigma) \varepsilon}^{\lambda, \mu^{\prime}}, \mathcal{R}_{Q}, \phi_{N^{\prime}}\right)$ so that $\delta d^{\prime \prime \prime}=\rho_{\mu, \mu^{\prime}}^{*}, d^{\prime \prime}$ and

$$
\left\|d^{\prime \prime \prime}\right\| \phi_{N^{\prime}} \leqq C_{2}\left\|d^{\prime \prime}\right\|_{\phi_{N}} \text {. }
$$

Setting $\gamma=\rho_{\mu, \mu^{\prime}}^{*} d^{\prime}-d^{\prime \prime \prime} \in C^{\sigma}\left(\mathcal{L}^{\left(\mu^{\prime}\right)} \cap \Omega_{(\tau-\sigma) \varepsilon}^{\lambda, \mu^{\prime}}, O^{r}, \phi_{N^{\prime}}\right)$, we have $\delta \gamma=\rho_{\mu, u^{\prime}}^{*} d^{\prime \prime}$ $-\delta d^{\prime \prime \prime}=0$ and

$$
\|\gamma\|_{\phi_{N^{\prime}}} \leqq C_{3}\|c\|_{\phi} .
$$

Hence Proposition 3. 1 shows that for some $" \ell^{\prime \prime}>\mu^{\prime}$ and $N^{\prime \prime}>N^{\prime}$ one can fined $\gamma^{\prime} \in C^{v-1}\left(\mathcal{U}^{\left(\mu^{\prime \prime}\right)} \cap \Omega_{(\tau-\sigma+1) \varepsilon}^{\lambda, \mu^{\prime \prime}}, \Theta^{r}, \phi_{N^{\prime \prime}}\right)$ so that $\rho_{\mu^{\prime}, \mu^{\prime \prime}}^{*} \gamma=\delta \gamma^{\prime}$ and

$$
\left\|\gamma^{\prime}\right\|_{\phi_{N^{\prime \prime}}} \leqq C_{4}\|\gamma\|_{\phi_{N^{\prime}}} \leqq C_{5}\|c\|_{\phi} \text {. }
$$

Here we used the fact that $\Omega_{(:-\sigma+1) \varepsilon}$ is a pseudoconvex domain contained in $\Omega_{(\tau-\sigma) \varepsilon}^{\lambda, \mu^{\prime}}$ as $\varepsilon \geqq \sqrt{2 n} 3^{1-\lambda}$. If we set $c^{\prime}=Q \gamma^{\prime}$, it follows that

$$
\begin{aligned}
& \delta c^{\prime}=Q \delta \gamma^{\prime}=Q \rho_{n^{\prime}, \mu^{\prime \prime}}^{*} \gamma=Q \rho_{n^{\prime}, u^{\prime \prime}}^{*} \rho_{n, n^{\prime}}^{*} d^{\prime}-\rho_{k^{\prime}, \mu^{\prime \prime}}^{*} Q d d^{\prime \prime \prime} \\
& =\rho_{\mu, \mu^{\prime \prime}}^{*} Q d^{\prime}=\rho_{\mu, \mu^{\prime \prime}}^{*} \rho_{\nu, \mu}^{*} c=\rho_{\nu, \mu^{\prime \prime}}^{*} c \text {. }
\end{aligned}
$$

Since (3.6) implies (3.5) for suitable $\%$ and $N$, the proposition is 
proved.

Proposition 3. 5. Let $\Omega^{\prime}$ be an open set which is strictly contained in a pseudoconvex domain $\Omega$ of $C^{n}\left(\operatorname{dist}\left(\Omega^{\prime}, \Omega^{c}\right) \geqq \delta>0\right)$. Given the system $P$ there is a constant $N$ such that, if $\phi$ is a plurisubharmonic function satisfying (3.4), then for all $u \in \mathcal{O}(\Omega)^{q}$ one can find $v \in \mathcal{O}\left(\Omega^{\prime}\right)^{q}$ with $P v=P u$ and

$$
\int_{\Omega^{\prime}}|v|^{2} e^{-\phi}\left(1+|z|^{2}\right)^{-N} d \lambda \leqq C \int_{\Omega}|P u|^{2} e^{-\phi} d \lambda
$$

where $C$ is a constant independent of $u$.

Proof. First, choose $\nu$ so that $\delta>\tau \varepsilon=2^{2 n} \sqrt{2 n} 3^{1-\nu}$. By Proposition 3.3 we can shoose $\nu<\mu$ so that there exists an element $u_{g} \in \mathcal{O}\left(U_{g}^{(\mu)}\right)^{q}$ such that $P u_{g}=P u$ in $U_{g}^{(\mu)} \subset U_{g^{\prime}}^{(\nu)} \subset \Omega$, and for some constants $C$ and $N$ independent of $u$ and $g \in I$

$$
\int_{U_{g}^{(\mu)}}\left|u_{g}\right|^{2} e^{-\phi}\left(1+|z|^{2}\right)^{-N} d \lambda \leqq C \int_{U_{g^{\prime}}^{(\nu)}}|P u|^{2} e^{-\phi} d \lambda
$$

where $g^{\prime}=\rho_{\nu, \mu} g$. Let $c_{g_{1} g_{2}}=u_{g_{1}}-u_{g_{2}}$. This defines a cocycle $c \in C^{1}\left(U^{(\mu)}\right.$ $\cap \Omega^{\nu, \mu}, \mathscr{R}_{P}, \phi_{N}$ ) and by (3.8) we obtain

$$
\|c\|_{\phi_{N}}^{2} \leqq C^{\prime} \int_{\Omega}|P u|^{2} e^{-\phi} d \lambda
$$

Proposition 3. 4 asserts that for some $\lambda>\mu$ and $N^{\prime}>N$ there exists a cochain $c^{\prime} \in C^{0}\left(\mathcal{U}^{(\lambda)} \cap \Omega^{\prime}, \mathscr{R}_{P}, \phi_{N^{\prime}}\right)$ such that $\delta c^{\prime}=\rho_{\mu, 2}^{*} c \mid \Omega^{\prime}$ and

$$
\left\|c^{\prime}\right\|_{\phi_{N^{\prime}}} \leqq C^{\prime \prime}\|c\|_{\phi_{N}} \text {. }
$$

Here we used the fact that $\Omega^{\prime}$ is contained in $\Omega_{\tau \varepsilon}^{\nu, \lambda}$ as $\delta>\tau \varepsilon$. This means that if we set $v=u_{\rho_{\mu, \nu} g}+c_{g}^{\prime}$ in $U_{g}^{(\lambda)} \cap \Omega^{\prime}$, we define uniquely an element $v \in \mathcal{O}\left(\Omega^{\prime}\right)^{q}$. Since $P c_{g}^{\prime}=0$, it follows that $P v=P u$, and from the estimates (3.8), (3.9) and (3.10) we obtain (3.7) with $N$ replaced by $N^{\prime}$.

\section{$\S$ 4. Soft Resolution of $\mathcal{Q}_{k, \ell}$}

In this section, we define the space $\mathcal{G}^{\prime}(\Omega)$ of rapidly decreasing distributions, and using this space we make a resolution of $\mathcal{Q}_{k, l}$, that is, 


$$
0 \rightarrow \mathcal{Q}_{k, l} \rightarrow \mathcal{G}_{(0,0)}^{\prime} \stackrel{\bar{\partial}}{\rightarrow} \mathcal{Q}_{(0,1)}^{\prime} \stackrel{\bar{\partial}}{\rightarrow} \ldots \stackrel{\bar{\partial}}{\rightarrow} \mathcal{G}_{(0, n)}^{\prime} \rightarrow 0
$$

Definition 4. 1. Let $\Omega$ be an open set in $Q^{k, l}$. We denote by $\mathcal{G}(\Omega)$ the inductive limit $\lim _{K \subset \Omega} \mathscr{F}_{c}(K)$ of $\mathscr{F}_{c}(K)$, where $K$ is a compact set in $\Omega$. We denote by $\mathcal{G}^{\prime}(\Omega)$ the dual space of $\mathcal{G}(\Omega)$.

Since the injection of $\mathcal{G}(\Omega)$ into $\mathscr{E}(\Omega)$ (Definition 2.13 of $[\mathrm{I}]$ ) is continuous and of dense range, $\mathscr{F}^{\prime}(\Omega)$ is a linear subspace of $\mathcal{G}^{\prime}(\Omega)$. Moreover, we have the following proposition.

Proposition 4.2. An element of $\mathcal{G}^{\prime}(\Omega)$ belongs to $\mathscr{F}^{\prime}(\Omega)$ if and only if it has a compact support.

Proof. Let $T \in \mathscr{F}^{\prime}(\Omega)$. By the definition of the topology of $\mathscr{F}(\Omega)$ (see Definition 2.13 of [I]), there are a compact set $K$ in $\Omega$, an integer $m \geqq 0$, and a constant $C>0$ such that for all $\phi \in \mathscr{F}(\Omega)$,

$$
|\langle T, \phi\rangle| \leqq C \sup _{|\alpha| \leqq m, x \in K \cap \boldsymbol{C}^{n}}\left|D^{\alpha} \phi(x)\right| e^{-|x| /(m+1)} .
$$

This implies immediately that $\langle T, \phi\rangle=0$ whenever the support of $\phi$ is contained in the complement of $K$, which means that supp $T \subset K$.

Conversely if $T$ is an element of $\mathcal{G}^{\prime}(\Omega)$ with the compact support $K$. Let $\alpha(x) \in \mathscr{F}_{c}(\Omega)$ be equal to one in some neighbourhood of $K$. Then $\langle T, \phi\rangle=\langle T, \alpha \phi\rangle$ and if $\phi_{\nu}$ converges to zero in $\mathcal{F}(\Omega), \alpha \phi_{\nu}$ converges to zero in $\mathcal{G}(\Omega)$. Therefore $\mathscr{E}(\Omega) \ni \phi \rightarrow\langle T, \phi\rangle$ is continuous, hence $T \in \mathscr{I}^{\prime}(\Omega)$.

Proposition 4. 3. If $\Omega$ is a bounded open set in $\mathbb{C}^{n}$ then $\mathcal{G}^{\prime}(\Omega)$ $=\mathscr{D}^{\prime}(\Omega)$.

Proof. It is obvious, since $\mathcal{G}(\Omega)=\mathscr{D}(\Omega)$.

Proposition 4. 4. Let $K$ be a compact subsel of $Q^{k, l}$ defined in Definition 2.1, and $\Omega$ be a neighbourhood of $K$. For $f \in \mathscr{F}^{\prime}(\Omega)$, define

$$
\widehat{f}(\zeta)=\left\langle f, e^{-i\langle x, \zeta\rangle}\right\rangle /(2 \pi)^{n}
$$


then $\hat{f}(\zeta)$ is analytic in $\left\{\zeta \in C^{2 n} ;|\operatorname{Im} \zeta|<\varepsilon\right\}$ for some $\varepsilon>0$ and there exists an $N$ satisfying $|\widehat{f}(\zeta)| \leqq C(1+|\zeta|)^{N}$ for $|\operatorname{Im} \zeta|<\varepsilon$. The equality

$$
\langle f, v\rangle=\int_{\boldsymbol{R}^{2 n}} \widehat{f}(\xi+i \eta) \widetilde{v}(\xi+i \eta) d \xi
$$

holds for $v \in \mathscr{F}_{c}(K)$ and $\eta \in K^{\circ}$ with $|\eta|<\varepsilon$.

Proof. By the definition of the topology of $\mathcal{F}(\Omega)$, there exists a seminorm $\|\cdot\|_{L, N, \varepsilon}$ satisfying $|\langle f, v\rangle| \leqq C\|v\|_{L, N, \varepsilon}$ for some constant $C$, where $\|v\|_{L, N, \varepsilon}=\sup _{x \in L \cap \boldsymbol{R}^{2 n,|\alpha| \leq N}}\left|D^{\alpha} f(x)\right| e^{-\varepsilon|x|}$ for the compact set $L$ in $\Omega$ and $\varepsilon>0, N>0$. If $|\operatorname{Im} \zeta|<\varepsilon$, then

$$
\begin{aligned}
\left\|e^{-i\langle x, \zeta\rangle}\right\|_{L, N, \varepsilon} & =\sup _{x \in L \cap \boldsymbol{R}^{2 n},|\alpha| \leqq N}\left|\zeta^{\alpha} e^{-i\langle x, \zeta\rangle}\right| e^{-\varepsilon|x|} \\
& \leqq \sup _{|\alpha| \leqq N}\left\{\left|\zeta^{\alpha}\right|\right\} \leqq(1+|\zeta|)^{N}<\infty .
\end{aligned}
$$

Hence $\widehat{f}(\zeta)=\left\langle f, e^{-i\langle x, \zeta\rangle}\right\rangle /(2 \pi)^{n}$ is analytic in $|\operatorname{Im} \zeta|<\varepsilon$ and satisfies $|\widehat{f}(\zeta)| \leqq C(1+|\zeta|)^{N}$. Since

$$
v(x)=\frac{1}{(2 \pi)^{n}} \int_{\boldsymbol{R}^{2 n}} e^{-i\langle x, \hat{\xi}+i \eta\rangle} \widetilde{v}(\xi+i \eta) d \xi
$$

by Proposition 2.11, and the Riemann sum converges with respect to the seminorm $\|\cdot\|_{L, N, \varepsilon}$, then

$$
\begin{aligned}
\langle f, v\rangle & =\left\langle f, \frac{1}{(2 \pi)^{n}} \int_{\boldsymbol{R}^{2 n}} e^{-i\langle x, \xi+i \eta\rangle} \widetilde{v}(\xi+i \eta) d \xi\right\rangle \\
& =\int_{\boldsymbol{R}^{2 n}} \widehat{f}(\xi+i \eta) \widetilde{v}(\xi+i \eta) d \xi
\end{aligned}
$$

Remark 4.5. The equality (4.2) holds when $v$ satisfies $\left|D^{\alpha} v(x)\right|$ $\leqq C e^{\delta|x|}$ for $|\alpha| \leqq N+3 n$ and $\delta>0$ such that $K_{\delta}^{\circ}$ has an element $\eta$ satisfying $|\eta|<\varepsilon$.

Let $\bar{\partial}$ be the Cauchy-Riemann operator defined by

$$
\begin{aligned}
\bar{\partial}_{p}: u & =\sum_{i_{1}<, \cdots,<i_{p}} u_{i_{1}, \cdots, i_{p}} d \bar{z}_{i_{1}} \wedge \cdots \wedge d \bar{z}_{i_{p}} \rightarrow \\
w & =\sum_{i_{1}<, \cdots,<i_{p}, j}\left(\partial u_{i_{1}, \cdots, i_{p}} / \partial \bar{z}_{j}\right) d \bar{z}_{j} \wedge d \bar{z}_{i_{1}} \wedge \cdots \wedge d \bar{z}_{i_{p}} .
\end{aligned}
$$


If we identify forms $u$ and $w$ with vector functions $\breve{u}$ and $\check{w}$ having $\left(\begin{array}{c}n \\ p\end{array}\right)$ and $\left(\begin{array}{c}n \\ p+1\end{array}\right)$ components respectively, $\bar{\partial}_{p}$ can be represented by $P_{p}(D)$ where $P_{p}(\zeta)$ is a $\left(\begin{array}{l}n \\ p\end{array}\right)-\left(\begin{array}{c}n \\ p+1\end{array}\right)$ matrix with polynomial entries, and $D=i \partial / \partial x$. It is known as the Koszul resolution that the following sequence is exact:

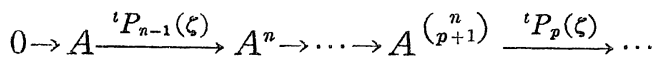

$$
\begin{aligned}
& \cdots \stackrel{{ }^{t} P_{0}(\zeta)}{\longrightarrow} A \rightarrow \operatorname{Coker}^{\imath} P_{0}(\zeta) \rightarrow 0,
\end{aligned}
$$

where $A$ is the polynomial ring of the variable $\zeta=\left(\zeta_{1}, \cdots, \zeta_{2 n}\right)$ and ${ }^{t} P_{p}(\zeta)$ is the transpose of $P_{p}(-\zeta)$ (see Example 4 in $\S 7$ of Chapter VII of V. P. Palamodov [5]). It is known that $\mathcal{R}_{t_{P_{p}}}$ is generated by the germs of the lows of the matrix $P_{p+1}(\zeta)$ (see Lemma 7.6.3 of L. Hörmander [1]). Since $\mathscr{R}_{\boldsymbol{t}_{\boldsymbol{p}}}$ is a coherent analytic sheaf, we have the following proposition.

Proposition 4. 6. Let $\Omega$ be a pseudoconvex domain. If $f \in \mathcal{O}^{r}(\Omega)$ satisfies the equation ${ }^{t} P_{p}(\zeta) f(\zeta)=0$, then therc exists a $g \in \mathcal{O}^{s}(\Omega)$ satisfying $f(\zeta)={ }^{t} P_{p+1}(\zeta) g(\zeta)$, where $r=\left(\begin{array}{c}n \\ p+1\end{array}\right)$ and $s=\left(\begin{array}{c}n \\ p+2\end{array}\right)$.

Proof. See Theorem 7.2.9 of L. Hörmander [1].

Definition 4. 7. (The sheaf of rapidly decreasing distributions.) We denote by $\mathcal{G}^{\prime}$ the sheaf determined by a presheaf $\left\{\mathcal{G}^{\prime}(\Omega)\right\}$, where $\Omega$ is an open set in $Q^{k, l}$.

For any locally finite covering $\left\{U_{\alpha}\right\}$ of $\Omega$, there exists a partition of unity $\left\{\phi_{\alpha}\right\}$ subordinate to the covering $\left\{U_{\alpha} \cap \mathbb{C}^{n}\right\}$ such that all derivatives of $\phi_{a}$ are bounded. Then $\mathcal{G}^{\prime}(\Omega)$ is the section module of the sheaf $\mathcal{G}^{\prime}$ and $\mathcal{G}^{\prime}$ is a soft sheaf.

Theorem 4. 8. Let $\Omega$ be a neighbourhood of a point $z_{\infty}$ at infinity in $Q^{k, l}$. If $f \in \mathcal{G}_{(0, p)}^{\prime}(\Omega)$ satisfies $\bar{\partial} f=0$, then there exists a neighbourhood $\omega$ of $\approx_{\infty}$ rith $\omega \subset \Omega$ and $u \in \mathcal{Q}_{(0, p-1)}^{\prime}(\omega)$ such that $\bar{\partial} u=f$ in $\omega$. 
Proof. First we choose a neighbourhood $\omega$ of $z_{\infty}$ having the form $\omega=a+$ Int $K$, where $K$ is the compact set in $\boldsymbol{Q}^{k, l}$ defined in Definition 2.1 and $a \in \boldsymbol{R}^{2 n}$.

Let $L$ be a compact set in $\Omega$ containing $\omega$. Then $f \in \mathscr{E}_{c}^{\prime}(L)^{J}$ and satisfies, for some $m>0, \varepsilon>0,|\langle f, \psi\rangle| \leqq C\|\psi\|_{m, \varepsilon}$ for all $\psi \in \mathscr{F}_{c}(L)^{J}$, where $J=\left(\begin{array}{l}n \\ p\end{array}\right)$. Hence

$$
|\langle f, \phi\rangle| \leqq C\|\phi\|_{m, \varepsilon} \quad \text { for } \quad \phi \in \mathcal{G}(\omega)^{J},
$$

where $\|\phi\|_{m, \varepsilon}=\sum_{j=1}^{J} \sup _{x \in \boldsymbol{R}^{2 n},|\alpha| \leqq m}\left|D^{\alpha} \phi_{j}(x)\right| e^{-\varepsilon|x|}$. If we can show that there exist $M>0$ and $\delta>0$ satisfying

$$
|\langle f, v\rangle| \leqq C\|\vartheta v\|_{M, \delta} \text { for all } v \in \mathcal{G}_{(0, n-p)}(\omega),
$$

by the Hahn-Banach theorem there exists a $u \in \mathcal{G}_{(0, p-1)}^{\prime}(\omega)$ satisfying $\langle f, v\rangle=\langle u, \vartheta v\rangle$, that is, $\bar{\partial} u=f$ in $\omega$, where $\vartheta$ is the dual operator of $\bar{\partial}$. Let $v \in \mathcal{G}(\omega)^{J}$, then supp $v \subset a+K$. By the coordinate transformation (translation) we may assume supp $v \subset K$. Then, by Corollary 2.8, $\widetilde{v}(\zeta)$ is analytic for $\operatorname{Im} \zeta \in K^{\circ}$ and satisfies, for any $\varepsilon>0$ and $\nu>0$,

$$
|\widetilde{v}(\zeta)| \leqq C_{\varepsilon, \nu} \frac{1}{(1+|\zeta|)^{\nu}} e^{h_{K, \varepsilon}(\operatorname{Im} \zeta)} \quad \text { for } \quad \operatorname{Im} \zeta \in K_{\varepsilon}^{\circ}
$$

Let $\bar{\partial}_{p}$ be represented by $P_{p}(D)$, then by Proposition 3.5 there exists an $N$ such that for any $\nu$ there exists a function $V(\zeta)$ analytic for $\operatorname{Im} \zeta \in \operatorname{Int} K_{2 \varepsilon}^{\circ}$ and satisfying

$$
{ }^{t} P_{p-1}(\zeta) V(\zeta)={ }^{t} P_{p-1}(\zeta) \widetilde{v}(\zeta)
$$

and

$$
\begin{aligned}
& \int_{R^{2 n}+i \operatorname{Int} K_{2 \varepsilon}^{\circ}}|V(\zeta)|^{2} e^{-2 h_{K, \varepsilon}(\operatorname{Im} \zeta)}\left(1+|\zeta|^{2}\right)^{\nu-N} d \lambda \\
\leqq & \int_{R^{2 n}+i K_{\varepsilon}^{\circ}}\left|{ }^{t} P_{p-1}(\zeta) \widetilde{v}(\zeta)\right|^{2} e^{-2 h_{K, \varepsilon}(\operatorname{Im} \xi)}\left(1+|\zeta|^{2}\right)^{\nu} d \lambda<\infty,
\end{aligned}
$$

where we have used the fact that $h_{K, \varepsilon}(\operatorname{Im} \zeta)$ is a convex (hence plurisubharmonic) function satisfying the condition (3.4) and $\boldsymbol{R}^{2 n}+i \operatorname{Int} K_{2 \varepsilon}^{\circ}$ is a pseudoconvex domain strictly contained in $\boldsymbol{R}^{2 n}+i$ Int $K_{\varepsilon}^{\circ}$ (see Propositions 2.15 and 2.16). From the above inequality, we have

$$
|V(\zeta)| \leqq C \frac{1}{(1+|\zeta|)^{\nu-N}} e^{h_{K, \varepsilon}(\operatorname{Im} \zeta)} \quad \text { for } \quad \operatorname{Im} \zeta \in K_{3 \varepsilon}^{\circ}
$$


Propositions 2.10,2.12 and the above inequality imply that $V(\zeta)=\widetilde{v}_{1}(\zeta)$ for a $C^{y-N-s n}$ function $v_{1}$ with support contained in $K$ satisfying $\left\|v_{1}\right\|_{\nu-N-3 n, 3 A \varepsilon}<\infty$. From Propositions 3.5 and 4.6, there exists a function $\Phi(\zeta)$ analytic in $\left\{\zeta \in \mathbb{C}^{2 n} ; \operatorname{Im} \zeta \in \operatorname{Int} K_{3 \varepsilon}^{\circ}\right\}$ and satisfying $V(\zeta)-\widetilde{v}(\zeta)$ $={ }^{t} P_{p}(\zeta) \Phi(\zeta)$ and

$$
\int_{R^{2 n} n_{\Gamma i K_{s c}^{0}}}|\Phi(\zeta)|^{2} e^{-2 h_{K, \varepsilon(\mathrm{Im} \zeta)}}\left(1+|\zeta|^{2}\right)^{\nu-N^{\prime \prime}} d \lambda<\infty,
$$

for some constant $N^{\prime}$ depending only on $P_{p}(\zeta)$ and $P_{p-1}(\zeta)$. This implies that there exists a $C^{\nu-N^{\prime}-8 n}$ function $\phi$ with support contained in $K$, satisfying $\Phi(\zeta)=\widetilde{\phi}(\zeta)$ and $\|\phi\|_{\nu-N^{\prime}-3 n, 4 A \varepsilon}<\infty$.

Considering the inequality (4.3), if we take sufficiently large $\nu>0$ and small $\varepsilon>0$, we have

$$
\left\langle f, v_{1}\right\rangle-\langle f, v\rangle=\left\langle f,{ }^{t} P_{p}(D) \phi\right\rangle=\left\langle P_{p}(D) f, \phi\right\rangle=\langle\bar{\partial} f, \phi\rangle=0 .
$$

Let $\alpha \in \mathscr{F}_{c}(L)$ with $\alpha(x)=1$ on a neighbourhood of $\omega \cap \mathbb{R}^{2 n}$. Define $f_{0}=\alpha f$, then $f_{0} \in \mathscr{F}^{\prime}(\Omega)$ by Proposition 4.2, and $\langle f, v\rangle=\left\langle f_{0}, v\right\rangle$ for any $C^{N}$ function $v$ with support contained in $\bar{\omega}$ and satisfying $\|v\|_{m, \varepsilon}<\infty$.

By Remark 4.5 if we take sufficiently large $\nu>0$ and small $\varepsilon>0$, we have

$$
\begin{aligned}
|\langle f, v\rangle|^{2}= & \left.i\left\langle f, v_{1}\right\rangle\right|^{2}=\mid\left\langle f_{0}, v_{1}\right\rangle^{2} \\
\leqq & \left(\int_{\boldsymbol{R}^{2 n}}\left|\hat{f}_{0}(\xi+i \eta) \widetilde{v}_{1}(\xi+i \eta)\right| d \xi\right)^{2} \\
\leqq & \int_{\boldsymbol{R}^{2 n}}\left|\hat{f}_{0}(\xi+i \eta)\right|^{2}\left(1+|\xi|^{2}\right)^{N-\nu} d \xi \\
& \times \int_{\boldsymbol{R}^{2 n}}|V(\xi+i \eta)|^{2}\left(1+|\xi|^{2}\right)^{\nu-N} d \xi \\
\leqq & C_{1} \int_{\boldsymbol{R}^{2 n}+i K_{2 c}^{\circ}}|V(\zeta)|^{2} e^{-2 h_{K, \varepsilon}(\operatorname{Im} \zeta)}\left(1+|\zeta|^{2}\right)^{\nu-N} d \lambda \\
\leqq & \int_{\boldsymbol{R}^{2 n}+i K_{\varepsilon}^{\circ}}\left|{ }^{t} P_{p-1}(\zeta) \widetilde{v}(\zeta)\right|^{2} e^{-2 h_{R, \varepsilon}(\operatorname{Im} \zeta)}\left(1+|\zeta|^{2}\right)^{\nu} d \lambda \\
\leqq & C\left\|^{t} P_{p-1}(D) v\right\|_{M, \delta}=C\|\vartheta v\|_{M, \delta} .
\end{aligned}
$$

The last inequality follows from Proposition 2.13. Thus we have shown (4.4), and completed the proof. 
Theorem 4. 9. We have the following soft resolution of the sheaf $\mathcal{O}_{k, l}$ :

$$
0 \rightarrow \mathcal{Q}_{k, l} \rightarrow \mathcal{G}_{(0,0)}^{\prime} \stackrel{\bar{\partial}}{\rightarrow} \mathcal{G}_{(0,1)}^{\prime} \stackrel{\bar{\partial}}{\rightarrow} \ldots \stackrel{\bar{\partial}}{\rightarrow} \mathcal{G}_{(0, n)}^{\prime} \rightarrow 0
$$

Proof. Since the restriction of $\mathcal{Q}_{k, l}$ or $\mathcal{G}^{\prime}$ to $\mathbb{C}^{n}$ is $\mathcal{O}$ or $\mathscr{D}^{\prime}$, respectively, and it is well known that the following sequence is exact:

$$
0 \rightarrow \Theta \rightarrow \mathscr{D}_{(0,0)}^{\prime} \stackrel{\bar{\partial}}{\rightarrow} \cdots \stackrel{\bar{\partial}}{\rightarrow} \mathscr{D}_{(0, n)}^{\prime} \rightarrow 0
$$

In order to obtain the resolution (4.5), we have only to make it at points at infinity. It is done in Theorem 4.8.

Definition 4. 10. Let $K$ be the compact set in $Q^{k, l}$ defined in Definition 2. 1. Define $I_{K, \varepsilon}(\eta)=\sup _{x \in K \cap \boldsymbol{R}^{2 n}}(\langle x, \eta\rangle-\varepsilon|x|)$ and $K_{(\varepsilon)}^{\circ}=\left\{\eta \in \boldsymbol{R}^{2 n}\right.$; $\left.I_{K, \varepsilon}(\eta)<\infty\right\}$.

Proposition 4. 11. Let $\Omega$ be an open set in $Q^{k, l}$ containing $K$. If $f \in \mathcal{F}^{\prime}(\Omega)$ satisfies the inequality $|\langle f, v\rangle| \leqq C\|v\|_{K, N, \varepsilon}$ for all $v \in \mathscr{I}(\Omega)$, where $\|v\|_{K, N, \varepsilon}=\sup _{x \in K \cap \boldsymbol{R}^{2 n},|\alpha| \leqq N}\left|D^{\alpha} f(x)\right| e^{-\varepsilon|x|}$, then $\hat{f}(\zeta)=$ $\left\langle f, e^{-i\langle x, \zeta\rangle}\right\rangle /(2 \pi)^{n}$ is analytic in $\left\{\zeta \in \mathscr{C}^{2 n} ; \operatorname{Im} \zeta \in \operatorname{Int} K_{(\varepsilon)}^{\circ}\right\}$ and satisfies, for some constant $C>0$,

$$
|\widehat{f}(\zeta)| \leqq C(1+|\zeta|)^{N} e^{I_{K, \varepsilon(\operatorname{Im} \zeta)}} \quad \text { for } \quad \operatorname{Im} \zeta \in K_{(\varepsilon)}^{\circ} .
$$

Proof. Let $\zeta=\xi+i \eta$ and $\eta \in K_{(\varepsilon)}^{\circ}$. Then we have

$$
\begin{aligned}
\left\|e^{-i\langle x, \zeta\rangle}\right\|_{K, N, \varepsilon} & =\sup _{x \in K \cap \boldsymbol{R}^{2 n},|\alpha| \leqq N}\left|\zeta^{\alpha} e^{\langle x, \eta\rangle}\right| e^{-\varepsilon|x|} \\
& \leqq(1+|\zeta|)^{N} e^{I_{K, \varepsilon}(\eta)}
\end{aligned}
$$

Since $\left(e^{-i\langle x, \zeta+h\rangle}-e^{-i\langle x, \zeta\rangle}\right) / h$ converges to $-i x e^{-i\langle x, \zeta\rangle}$ as $h \rightarrow 0$ with respect to $\|\cdot\|_{K, N, \varepsilon}$ for $\operatorname{Im} \zeta \in \operatorname{Int} K_{(\varepsilon)}^{\circ}, \widehat{f}(\zeta)$ is analytic.

Proposition 4. 12. Let $F(\zeta)$ be an analytic function in $\left\{\zeta \in \mathbb{C}^{2 n}\right.$; $\left.\operatorname{Im} \zeta \in \operatorname{Int} K_{(\varepsilon)}^{\circ}\right\}$ satisfying the inequality (4.6). Then $F(\zeta)$ defines an element $f \in \mathscr{F}^{\prime}\left(\mathbb{Q}^{k, l}\right)$ with support contained in $K$ satisfying

$$
\langle f, \phi\rangle=\int_{\boldsymbol{R}^{2 n}+i \eta} F(\zeta) \widetilde{\phi}(\zeta) d \zeta \quad \text { for } \quad \phi \in C_{0}^{\infty}\left(\boldsymbol{R}^{2 n}\right) .
$$


Proof. If $\phi \in C_{0}^{\infty}\left(\mathbb{R}^{2 n}\right)$, then $\widetilde{\phi}(\zeta)$ is an entire function satisfying for any $\nu>0$

$$
|\widetilde{\phi}(\zeta)|<C e^{h_{B}(\operatorname{Im} \zeta)} /(1+|\zeta|)^{\nu},
$$

where $B$ is the support of $\phi$ and $h_{B}(\eta)=\sup _{x \in B}(-\langle x, \eta\rangle)$. Hence the linear form

$$
\int_{\mathbb{R}^{2 n}+i \eta} F(\zeta) \widetilde{\phi}(\zeta) d \zeta=\langle f, \phi\rangle
$$

defines a distribution $f$. Let $B$ be convex and $B \cap K=\phi$, then therc exists a vector $\eta \in\left(-K^{\circ}\right) \subset K_{(\varepsilon)}^{\circ}$ such that for some $\delta>0$

$$
\sup _{x \equiv K \cap \boldsymbol{R}^{2 n}}\langle x, \eta\rangle \leqq\langle y, \eta\rangle-\hat{o}|\eta| \quad \text { for all } \quad y \in B
$$

hence $I_{K, \varepsilon}(\eta)+h_{B}(\eta) \leqq-\delta|\eta|$. Thus we have

$$
\begin{aligned}
\lim _{t \rightarrow \infty} & \int_{\boldsymbol{R}^{: n}+i t \eta} F(\zeta) \widetilde{\phi}(\zeta) d \zeta \\
& \leqq \lim _{t \rightarrow \infty} C c^{I_{K, \varepsilon}(t \eta)+h_{B}(t \eta)} \leqq \lim _{t \rightarrow \infty} C e^{-t \delta|\eta|}=0
\end{aligned}
$$

Hence the support of $f$ is contained in $K$. Let $L$ be a neighbourhood of $K$ having the form of Definition 2.1. If $\psi \in \mathcal{F}_{c}(L)$ then $\widetilde{\psi}(\zeta)$ is analytic in $\left\{\zeta \in \mathbb{C}^{2 n} ; \operatorname{Im} \zeta \in L^{\circ}\right\}$ and satisfies for any $\nu>0$ and $\varepsilon>0$

$$
|\widetilde{\psi}(\zeta)| \leqq C e^{h_{L, \varepsilon}(\operatorname{Im} \zeta)} /(1+|\zeta|)^{\nu} \quad \text { for } \quad \operatorname{Im} \zeta \in L_{\varepsilon}^{\circ} \text {. }
$$

Hence it follows from the formula

$$
\int_{\boldsymbol{R}^{2 n}+i \eta} F(\zeta) \widetilde{\psi}(\zeta) d \zeta
$$

that the distribution $f$ can be extended to $\mathscr{I}_{c}(L)$. Let $\alpha \in \mathscr{F}_{c}(L)$ such that $\alpha(x)=1$ in a neighbourhood of $K$, then $\alpha v \in \mathscr{F}_{c}(L)$ for $v \in \mathscr{F}\left(\mathbb{Q}^{k, l}\right)$. Since the support of $f$ is contained in $K$, we have $\langle f, v\rangle=\langle f, \alpha v\rangle$. This shows that $f \in \mathscr{E}^{\prime}\left(\mathbb{Q}^{k, l}\right)$.

Let $\Omega$ be an open set in $Q^{k, l}$ which has the form $a+\operatorname{Int} K$, wherc $K$ is the convex set defined in Definition 2.1 and $a \in \mathbb{C}^{n}$.

Theorem 4. 13. If $\bar{\partial}_{p} v=0$ for $v \in \Phi_{(0, \rho)}(\Omega)$, then there exists $u \in \mathcal{E}_{(0, p-1)}(\Omega)$ satisfying $\bar{\partial}_{p-1} u=v$. 
Proof. We represent $\bar{\partial}_{p}$ by $P_{p}(D)$. Since all the spaces of the sequence

$$
\mathscr{F}(\Omega)^{q} \stackrel{P_{p-1}(D)}{\longrightarrow} \mathscr{E}(\Omega)^{r} \stackrel{P_{p}(D)}{\longrightarrow} \mathscr{I}(\Omega)^{s}
$$

are FS spaces (see Remark 2.27 in [I]), we have only to show that the dual sequence

$$
\mathscr{E}^{\prime}(\Omega)^{q} \stackrel{t P_{p-1}(D)}{\longleftarrow} \mathscr{I}^{\prime}(\Omega)^{r} \stackrel{t P_{p}(D)}{\longleftarrow} \mathscr{F}^{\prime}(\Omega)^{s}
$$

is exact and the range of ${ }^{t} P_{p-1}(D)$ is closed.

Let $g \in \mathscr{F}^{\prime}(\Omega)^{r}$, then there exist a convex set of the form $b+L$ contained in $\Omega$ and constants $N>0, \varepsilon>0$ such that the estimate

$$
|\langle g, v\rangle| \leqq C\|v\|_{b+L, N, \varepsilon}
$$

holds for all $v \in \mathscr{E}(\Omega)^{r}$. We may assume that $L$ is also a convex set of the type in Definition 2.1. By coordinate transformation (translation) we may also assume $b=0$. Then, by Proposition 4.11, $\hat{g}(\zeta)$ is analytic in $\left\{\zeta \in C^{2 n} ; \operatorname{Im} \zeta \in \operatorname{Int} L_{(\varepsilon)}^{\circ}\right\}$ and satisfies

$$
|\widehat{g}(\zeta)| \leqq C(1+|\zeta|)^{N} e^{I_{L, \varepsilon(\operatorname{Im} \xi)}} \quad \text { for } \quad \operatorname{Im} \zeta \in L_{(\varepsilon)}^{\circ} .
$$

The equation ${ }^{t} P_{p-1}(D) g=0$ implies ${ }^{t} P_{p-1}(-\zeta) \widehat{g}(\zeta)=0$ in $\left\{\zeta \in \boldsymbol{C}^{2 n} ; \operatorname{Im} \zeta\right.$ $\in$ Int $\left.L_{(\varepsilon)}^{\circ}\right\}$. Then by Propositions 3.5 and 4.6 , there exists an analytic function $F(\zeta)$ such that ${ }^{t} P_{p}(-\zeta) F(\zeta)=\widehat{g}(\zeta)$ for $\operatorname{Im} \zeta \in \operatorname{Int} L_{(\varepsilon / 2)}^{\circ}$ and satisfying for some $\nu>0$

$$
|F(\zeta)| \leqq C(1+|\zeta|)^{\nu} e^{I_{L, \varepsilon / 2}(\operatorname{Im} \zeta)} \quad \text { for } \quad \operatorname{Im} \zeta \in L_{(\varepsilon / 2)}^{0} .
$$

Here we used the fact that $I_{L, \varepsilon}(\eta)$ is convex and Lipschitz continuous, and $L_{(\varepsilon / 2)}^{\circ}$ is a convex set contained strictly in $L_{(\varepsilon)}^{\circ}$. This shows that there exists $f \in \mathscr{F}^{\prime}(\Omega)^{s}$ such that

$$
\begin{aligned}
\left\langle f, P_{k}(D) v\right\rangle & =\int_{\boldsymbol{R}^{2 n}+i \eta} F(\zeta) P_{k}(\zeta) \widetilde{v}(\zeta) d \zeta \\
& =\int_{\boldsymbol{R}^{2 n}+i \eta}{ }^{t} P_{k}(-\zeta) F(\zeta) \widetilde{v}(\zeta) d \zeta \\
& =\int_{\boldsymbol{R}^{2 n}+i \eta} \widehat{g}(\zeta) \widetilde{v}(\zeta) d \zeta=\langle g, v\rangle
\end{aligned}
$$

for all $v \in \mathscr{F}_{c}(K)$, that is, ${ }^{t} P_{k}(D) f=g$.

Next we prove the closedness of the range of ${ }^{t} P_{0}(D)$. Assume 
$F_{j} \rightarrow F$ in $\mathscr{E}^{\prime}(\Omega)$ with $F_{j}={ }^{t} P_{0}(D) G_{j}$ for $G_{j} \in \mathscr{F}^{\prime}(\Omega)^{n}$. Since the sequence $\left\{F_{j}\right\}$ is a bounded set in the $D F S$ space $\mathscr{F}^{\prime}(\Omega)$, there exist a compact set $L$ in $\Omega$ (we may assume that $L$ is a convex set of the type in Definition 2.1) and constants $C>0, \varepsilon>0$ satisfying

$$
\left|\bar{F}_{j}(\zeta)\right| \leqq C(1+|\zeta|)^{N} e^{I_{L, \varepsilon}(\operatorname{Im} \tilde{)})} \quad \text { for } \quad \operatorname{Im} \zeta \in L_{(\epsilon)}^{\circ} .
$$

By Proposition 3.5 we can choose $\Psi_{j}(\zeta)$ satisfying

$$
\left|\Psi_{j}(\zeta)\right| \leqq C^{\prime}(1+|\zeta|)^{N^{\prime}} e^{I_{L, \varepsilon(\operatorname{Im} \zeta)}} \quad \text { for } \quad \operatorname{Im} \zeta \in L_{(\varepsilon / 2)}^{(0}
$$

Since $\left\{\Psi_{j}(\zeta)\right\}$ forms a normal family, there exists a subsequence which converges to $\Psi(\zeta)$ which also satisfies (4.8). Thus there exists $G \in \mathcal{F}^{\prime}(\Omega)^{n}$ satisfying

$$
\begin{aligned}
\left\langle G, P_{0}(D) v\right\rangle & =\int_{\boldsymbol{R}^{2 n+i \eta}} \Psi(\zeta) P_{0}(\zeta) \widetilde{v}(\zeta) d \zeta \\
& =\lim _{k \rightarrow \infty} \int_{\boldsymbol{R}^{2 n}+i \eta}{ }^{t} P_{0}(-\zeta) \Psi_{j_{k}}(\zeta) \widetilde{v}(\zeta) d \zeta \\
& =\lim _{k \rightarrow \infty}\left\langle F_{j_{k}}, v\right\rangle=\langle F, v\rangle .
\end{aligned}
$$

This shows that $F={ }^{t} P_{0}(D) G$, that is, the range of ${ }^{t} P_{0}(D)$ is closed.

At the end of this section, we give an extension of Theorem 4.11 of [I].

Theorem 4. 14. We have the follorving soft resolution of the sheaf $\widetilde{\mathcal{O}}_{k, l}$ on $Q^{k, l}$ :

$$
0 \rightarrow \widetilde{\mathcal{O}}_{k, l} \rightarrow \mathscr{E}_{(0,0)} \stackrel{\bar{\partial}}{\rightarrow} \cdots \stackrel{\bar{\partial}}{\rightarrow} \mathscr{E}_{(0, n)} \rightarrow 0
$$

Proof. Since the restriction of $\widetilde{\mathcal{O}}_{k, l}$ or $\mathscr{I}$ to $C^{n}$ is $\Theta$ or $\mathcal{E}$ respectively, and it is well known that the following sequence is exact:

$$
0 \rightarrow \mathcal{O} \rightarrow \mathcal{E}_{(0,0)} \stackrel{\bar{\partial}}{\rightarrow} \cdots \stackrel{\bar{\partial}}{\rightarrow} \mathcal{E}_{(0, n)} \rightarrow 0
$$

In order to obtain the resolution (4.7) of $\widetilde{\mathcal{O}}_{k, l}$, we have only to make the resolution at points at infinity. Since the point $z_{\infty}$ at infinity has a fundamental system of neighbourhoods whose nember has the form $a+$ Int $K$, Theorem 4.13 gives the resolution at points at infinity. 
Remark 4.15. In the above theorem the resolution is obtained on the whole $\boldsymbol{Q}^{k, l}$, while in Theorem 4.11 of [I], it is obtained on the open subset $\Omega$ which satisfies the condition (i) of Definition 4.5 of [I].

\section{§ 5. Fourier Hyperfunctions with Compact Supports}

In this section, we show that the space $H_{K}^{n}\left(V,{ }^{E} \widetilde{\mathcal{O}}_{k, l}\right)$ of $E$-valued Fourier hyperfunctions is isomorphic to the space $L\left(\mathcal{Q}_{k, l}(K), E\right)$ of continuous linear mappings from $\mathcal{Q}_{k, l}(K)$ to a Fréchet space $E$.

Let $K$ be a compact set in $\prod_{i=1}^{j} \mathbb{D}^{n_{i}}$ and $V$ be an $\widetilde{\mathcal{O}}_{k, l}$-pseudoconvex neighbourhood of $K$ in $\mathbb{Q}^{k, l}$. From Theorem 5.8 and Corollary 5.10 of $[I]$, we have $H_{c}^{p}\left(V, \mathcal{O}_{k, l}\right)=0$ for $0 \leqq p \leqq n-1$ and $H^{p}\left(K,{\underset{\sim}{k, l}}_{k}\right)=0$ for $p \geqq 1$. Therefore from the long exact sequence of cohomology groups with compact supports,

$$
\begin{aligned}
& 0 \rightarrow H_{c}^{0}\left(V-K, \mathcal{O}_{k, l}\right) \rightarrow H_{c}^{0}\left(V, \mathcal{\sim}_{k, l}\right) \rightarrow H^{0}\left(K, \mathcal{\sim}_{k, l}\right) \\
& \stackrel{\delta}{\rightarrow} H_{c}^{1}\left(V-K, \mathcal{Q}_{k, l}\right) \stackrel{\rho}{\rightarrow} H_{c}^{1}\left(V, \mathcal{\sim}_{k, l}\right) \rightarrow H^{1}\left(K, \mathcal{\sim}_{k, l}\right) \\
& \rightarrow H_{c}^{2}\left(V-K, \underset{\sim}{\mathcal{O}_{k, l}}\right) \rightarrow H_{c}^{2}\left(V, \mathcal{O}_{k, l}\right) \rightarrow \cdots,
\end{aligned}
$$

follows that $\delta: H^{0}\left(K,{\underset{\sim}{\mathcal{O}}}_{k, l}\right) \cong H_{c}^{1}\left(V-K, \underset{\sim}{\mathcal{Q}_{k, l}}\right)$ and $H_{c}^{2}\left(V-K, \underset{\mathcal{O}_{k, l}}{ }\right)=0$, for $n \geqq 2$.

Since by Theorem 4.9 we have the soft resolution

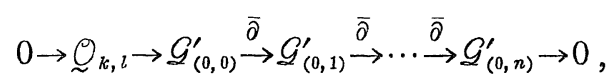

$H_{c}^{1}\left(V-K, \underset{\sim}{\mathcal{Q}_{k, l}}\right)$ can be represented by the first cohomology group of the complex $\left.\left(\mathscr{E}^{\prime}{ }_{(0 .)}\right)(V-K), \bar{\partial}\right)$. Then $\delta$ can be represented as the following continuous mapping. Let $U$ be an open neighbourhood of $K$ and $\alpha \in \mathscr{F}_{c}(U)$ such that $\alpha=1$ in $W \cap \boldsymbol{R}^{2 n}$, where $W$ is some neighbourhood of $K$ in $U$. The map

$$
\delta_{U, a}: H^{0}\left(U, Q_{k, l}\right) \rightarrow\left\{u \in \mathscr{F}_{(0,1)}^{\prime}(V-K) ; \bar{\partial} u=0\right\}
$$

defined by $\delta_{U, \alpha}(f)=\bar{\partial}(\alpha f)$ is continuous and induces a continuous map of $H^{0}\left(U, \mathcal{Q}_{k, l}\right)$ into $H_{c}^{1}\left(V-K, \mathcal{Q}_{k, l}\right)$. These maps define the map $\delta$ on

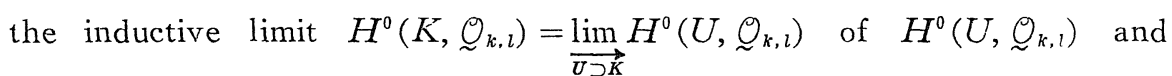
therefore $\delta$ is continuous. Moreover we can show that $\delta$ is an open mapping. 
Proposition 5. 1. Let $n \geqq 2$. Consider the dual complex,

$$
\begin{gathered}
\rightarrow \mathscr{F}_{(0, n-2)}(V-K) \stackrel{\bar{\partial}_{n-2}}{\longrightarrow} \mathscr{F}_{(0, n-1)}(V-K) \stackrel{\bar{\partial}_{n-1}}{\longrightarrow} \mathscr{F}_{(0, n)}(V-K) \rightarrow 0 \\
\uparrow \\
\leftarrow \mathcal{F}_{(0,2)}^{\prime}(V-K) \stackrel{-\bar{\partial}_{1}}{\longleftarrow} \mathscr{F}_{(0,1)}^{\prime}(V-K) \stackrel{-\bar{\partial}_{0}}{\longleftarrow} \mathcal{F}_{(0,0)}^{\prime}(V-K) \leftarrow 0 .
\end{gathered}
$$

Then the ranges of the operators are all closed.

Proof: $H_{c}^{2}\left(V-K, \underset{\sim}{\mathcal{O}_{k, l}}\right)=0$ shows that the range of $-\bar{\partial}_{1}$ is closed, and from Theorem 5.11 of [I], it follows that the range of $\bar{\partial}_{n-1}$ is closed. The closedness of ranges of other operators is a consequence of the so-called Serre-Komatsu duality theorem (see Theorem 4.7 of [I]).

Proposition 5.2. Let $n \geqq 2$, then $H^{0}\left(K,{\underset{\sim}{N, l}}_{k}\right)$ and $H_{c}^{1}\left(V-K, \mathcal{O}_{k, l}\right)$ are DFS spaces.

Proof. Proposition 2.7 of [I] shows that $H^{0}\left(K, \underset{\mathcal{O}_{k, l}}{\mathcal{1}}\right)=\mathcal{Q}_{k, l}(K)$ is a $D F S$ space. $\mathscr{F}_{(0,1)}^{\prime}(V-K)$ is a $D F S$ space as the dual space of an $F S$ space $\mathscr{I}_{(0,1)}(T-K)$ (see Remark 2.27 of [I]). Since a closed subspace and a quotient space (by its closed subspace) of a $D F S$ space are also $D F S$ spaces, it follows from the fact that the range of $-\bar{\partial}_{0}$ is closed, that $H_{c}^{1}\left(V-K, \mathcal{O}_{k, l}\right)$ is a $D F S$ space.

Theorem 5. 3. Let $E$ be a fully complete space, and let $F$ be a barrelled space. Let $f$ be a linear mapping of a subspace $E_{0} \subset E$ onto $F$. Suppose thal the graph of $f$ is closed in $E \times F$. Then $f$ is open.

Proof. See Theorem 4.10 of V. Pták [6].

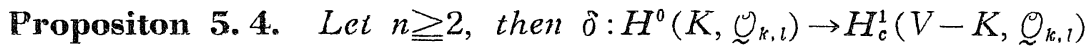
is a homeomorphism.

Proof. It is known that DFS spaces are fully complete and barrelled spaces (see Theorems 4.3.28 and 4.3.40 of H. Komatsu [3]). Since $\hat{o}$ is a one-to-one onto continuous mapping, it follows from Theorem 
5. 3 that $\delta$ is a homeomorphism.

Proposition 5. 5. Let $n \geqq 2$, then $H^{n-1}\left(V-K, \widetilde{\mathcal{O}}_{k, l}\right) \cong\left[\mathcal{Q}_{k, l}(K)\right]^{\prime}$.

Proof. $\quad H^{n-1}\left(V-K, \widetilde{\mathcal{O}}_{k, 1}\right)$ is represented by the $(n-1)$-th cohomology group of the complex $\left(\mathscr{F}_{(0, .)}(V-K), \bar{\partial}\right)$. It follows from Proposition 5.1 and the so-called Serre-Komatsu duality theorem (Theorem 4. 7 of [I]) that

$$
H^{n-1}\left(V-K, \widetilde{\mathcal{O}}_{k, l}\right) \cong\left[H_{c}^{1}\left(V-K, \mathcal{O}_{k, l}\right)\right]^{\prime} \cong\left[\mathcal{\Theta}_{k, l}(K)\right]^{\prime} .
$$

Let $E$ be a Fréchet space. From the exact sequence,

$$
\begin{aligned}
\cdots \rightarrow & H_{K}^{p}\left(V,{ }^{E} \widetilde{\mathscr{O}}_{k, l}\right) \rightarrow H^{p}\left(V,{ }^{E} \widetilde{\mathcal{O}}_{k, l}\right) \\
& \rightarrow H^{p}\left(V-K,{ }^{E} \widetilde{\mathscr{O}}_{k, l}\right) \rightarrow H_{K}^{p+1}\left(V,{ }^{E} \widetilde{\mathscr{O}}_{k, l}\right) \rightarrow \cdots
\end{aligned}
$$

and the fact that if $V$ is $\widetilde{\mathcal{O}}_{k, l}$-pseudoconvex, $H^{p}\left(V,{ }^{E} \widetilde{\mathcal{O}}_{k, l}\right)=0$ for $p>0$ (see Theorem 6.6 of $[\mathrm{I}])$, it follows that $H_{K}^{n}\left(V,{ }^{E} \widetilde{\mathcal{O}}_{k, l}\right) \cong H^{n-1}\left(V-K,{ }^{E} \widetilde{\mathcal{O}}_{k, l}\right)$, for $n \geqq 2$.

Proposition 5. 6. Let $n \geqq 2$, then $H^{n-1}\left(V-K,{ }^{E} \widetilde{\mathcal{O}}_{k, l}\right) \cong H^{n-1}(V-K$, $\left.\widetilde{\mathcal{O}}_{k, l}\right) \hat{\otimes} E$ for a Fréchet space $E$.

Proof. We represent $H^{n-1}\left(V-K, \widetilde{\mathcal{O}}_{k, l}\right)$ by the $(n-1)$-th cohomology group of the complex,

$$
\cdots \rightarrow \mathscr{E}_{(0, n-2)}(V-K) \stackrel{\bar{\partial}_{n-2}}{\longrightarrow} \mathscr{I}_{(0, n-1)}(V-K) \stackrel{\bar{\partial}_{n-1}}{\longrightarrow} \mathscr{F}_{(0, n)}(V-K) \rightarrow 0 .
$$

Since the range of $\bar{\partial}_{n-2}$ is closed by Proposition 5.1 and $\mathcal{E}_{(0, n-1)}(V-K)$ is a Frécet nuclear space, we have the exact sequence

$$
0 \rightarrow \operatorname{jm} \bar{\partial}_{n-2} \rightarrow \operatorname{ker} \bar{\partial}_{n-1} \rightarrow \operatorname{ker} \bar{\partial}_{n-1} / \operatorname{im} \bar{\partial}_{n-2} \rightarrow 0
$$

where all the spaces are Fréchet nuclear spaces. Since the tensoring by $\hat{\otimes} E$ is a exact functor (see Theorem 6.5 of [I]), we have the following exact sequence:

$$
0 \rightarrow\left(\operatorname{im} \bar{\partial}_{n-2}\right) \hat{\otimes} E \rightarrow\left(\operatorname{ker} \bar{\partial}_{n-1}\right) \hat{\otimes} E \rightarrow H^{n-1}\left(V-K, \widetilde{\mathcal{O}}_{k, l}\right) \hat{\otimes} E \rightarrow 0 .
$$

If we denote the closed linear hull by [] , we have 


$$
\begin{aligned}
\operatorname{ker}\left(\bar{\partial}_{n-1} \hat{\otimes} 1_{E}\right) & =\left[f \otimes e \in \mathscr{I}_{(0, n-1)}(V-K) \hat{\otimes} E ; \bar{\partial}_{n-1} f=0\right] \\
& =\left(\operatorname{ker} \bar{\partial}_{n-1}\right) \hat{\otimes} E .
\end{aligned}
$$

By Proposition 43.9 of F. Treves [7], we also have im $\left(\bar{\partial}_{n-2} \otimes \grave{\otimes} 1_{E}\right)$ $=\left(\operatorname{im} \bar{\partial}_{n-2}\right) \hat{\hat{\otimes}} E$. Since $H^{n-1}\left(V-K,{ }^{E} \widetilde{\mathcal{O}}_{k, l}\right)$ can be represented by the $(n-1)$-th cohomology group of the complex $\left(\mathscr{F}_{(0,0)}(V-K, E),{ }^{E} \bar{\partial}\right)$ and $\mathscr{F}_{(0, \cdot)}(V-K, E) \cong \mathscr{F}_{(0, \cdot)}(V-K) \hat{\otimes} E$ and ${ }^{E} \bar{\partial}=\bar{\partial} \hat{\otimes} 1_{E}$, we have $H^{n-1}(V-K$, $\left.{ }^{E} \widetilde{\mathcal{O}}_{k, l}\right) \cong H^{n-1}\left(V-K, \widetilde{\mathcal{O}}_{k, l}\right) \hat{\otimes} E$.

Theorem 5. 7. Let $E$ be a Fréchet space and $K$ be a compact set in $\prod_{i=1}^{j} \boldsymbol{D}^{n_{1}}$. Then $H_{K}^{n}\left(V,{ }^{E} \widetilde{\mathcal{O}}_{k, l}\right) \cong L\left(\mathcal{O}_{k, l}(K), E\right)$.

Proof. By Proposition 50.5 of F. Treves [7], we have $L\left(\mathcal{Q}_{k, l}(K)\right.$, $E) \cong\left[\Theta_{k, l}(K)\right]^{\prime} \hat{\otimes} E$. Propositions 5.5 and 5.6 show that $\left[\mathcal{\sim}_{k, l}(K)\right]^{\prime}$ $\hat{\otimes} E \cong H^{n-1}\left(V-K,{ }^{E} \widetilde{\mathcal{O}}_{k, l}\right)$, for $n \geqq 2$. Thus we have $H_{K}^{n}\left(V,{ }^{E} \widetilde{\mathcal{O}}_{k, l}\right)$ $\cong L\left(\mathcal{Q}_{k, l}(K), E\right)$, for $n \geqq 2$.

If $n=1, H^{1}\left(W, \widetilde{\mathcal{O}}_{k, l}\right)=0$ for any open set $W$ in $Q^{k, l}$ satisfying the condition (i) of Definition 5.1 of [I] (Theorem 5.11 of [I]). Consider the dual complex,

$$
\begin{gathered}
0 \rightarrow \mathscr{F}_{(0,0)}(W) \stackrel{\bar{\partial}}{\longrightarrow} \mathscr{F}_{(0,1)}(W) \rightarrow 0 \\
\hat{\imath} \\
0 \leftarrow \mathscr{\Psi}_{(0,1)}^{\prime}(W) \stackrel{-\bar{\partial}}{\longleftarrow} \mathscr{\Psi}_{(0,0)}^{\prime}(W) \leftarrow-0 .
\end{gathered}
$$

Then the range of $\bar{\partial}\left(=\mathscr{F}_{(0,1)}(W)\right)$ is closed, therefore the range of $(-\bar{\partial})$ is closed and

$$
\widetilde{\mathcal{O}}_{k, l}(W) \cong\left[H_{c}^{1}\left(W, \mathcal{O}_{k, l}\right)\right]^{\prime} .
$$

The mapping $\rho$ of the exact sequence

$$
0 \rightarrow H^{0}\left(K, \mathcal{O}_{k, l}\right) \stackrel{\delta}{\rightarrow} H_{c}^{1}\left(V-K, \mathcal{Q}_{k, l}\right) \stackrel{\rho}{\rightarrow} H_{c}^{1}\left(V, \mathcal{Q}_{k, l}\right) \rightarrow 0
$$

is continuous since it is induced by the continuous injection of $\mathscr{F}^{\prime}(V-K)$ into $\mathscr{L}^{\prime}(V)$. Therefore the dual sequence

$$
0 \rightarrow \widetilde{\mathcal{O}}_{k, l}(V) \stackrel{\rho^{*}}{\rightarrow} \widetilde{\mathcal{O}}_{k, l}(V-K) \stackrel{\delta^{*}}{\rightarrow}\left[\mathcal{\sim}_{k, l}(K)\right]^{\prime} \rightarrow 0
$$

is exact. Since all the spaces of the above sequence are Fréchet nuclear, we have the exact sequence 


$$
0 \rightarrow \widetilde{\Theta}_{k, l}(V, E) \rightarrow \widetilde{\mathcal{O}}_{k, l}(V-K, E) \rightarrow\left[\mathcal{Q}_{k, l}(K)\right]^{\prime} \hat{\otimes} E \rightarrow 0,
$$

where we used the fact that $\widetilde{\mathcal{O}}_{k, l}(W, E) \cong \widetilde{\mathcal{O}}_{k, l}(W) \hat{\otimes} E$ for an open set $I^{r}$ in $\boldsymbol{Q}^{k, l}((6.6)$ of $[\mathrm{I}])$ and the tensoring $\hat{\otimes} E$ is an exact functor (Theorem 6.5 of $[\mathrm{I}]$ ). Thus we have

$$
\begin{aligned}
H_{K}^{n}\left(V,{ }^{E} \widetilde{\mathcal{O}}_{k, l}\right) & \cong \widetilde{\mathcal{O}}_{k, l}(V-K, E) / \widetilde{\mathcal{O}}_{k, l}(V, E) \cong\left[\mathcal{Q}_{k, l}(K)\right]^{\prime} \hat{\otimes} E \\
& \cong L\left(\bigodot_{k, l}(K), E\right),
\end{aligned}
$$

for $n=1$.

Corollary 5.8. Let $\Omega$ be an open set in $\prod_{i=1}^{j} \boldsymbol{D}^{n_{i}}$. Then ${ }^{E} \mathcal{R}_{k, l}(\Omega)$ $\cong L\left(\mathcal{Q}_{k, l}(\Omega), E\right) / L\left(\mathcal{Q}_{k l}(\partial \Omega), E\right)$.

Proof. The corollary follows from Proposition 6.10 of [I] and Theorem 5. 7.

Without changing the proof of Theorem 5.7, we can prove the following theorem, which corresponds to Theorem 5.12 of [I] in the scalar valued case.

Theorem 5. 9. Let $K$ be a compact set in $\boldsymbol{Q}^{k, l}$, and $V$ be an $\widetilde{\mathcal{O}}_{k, l}$-pseudoconvex domain containing $K$. Suppose $H^{p}\left(K, \mathcal{O}_{k, l}\right)=0$ for $p \geqq 1$. Then we have

$$
H_{K}^{n}\left(V,{ }^{E} \widetilde{\mathcal{O}}_{k, l}\right) \cong L\left(\mathcal{Q}_{k, l}(K), E\right)
$$

Remark 5. 10. We can also prove $H_{K}^{p}\left(V,{ }^{E} \widetilde{\widetilde{O}}_{k, l}\right)=0$ for $p \neq n$, for a compact set $K$ satisfying the condition of the above theorem, in the same way as Theorem 6.8 of [I].

\section{References}

[1] Hörmander, L., Complex Analysis in Several Variables, North-Holland, AmsterdamLondon, 1973.

[2] Komatsu, H., Projective and injective limits of weakly compact sequences of locally convex spaces, J. Math. Soc. Japan, 19 (1967), 366-383.

[3] - Satō no chökansū to teisū keisū senkei henbibun höteishiki, Seminar Note Dept, of Math. Tokyo Univ., 22, 1968 (in Japanese). 
[ 4] (=[I]) Nagamachi, S., The theory of vector valued Fourier hyperfunctions of mixed type. I, Publ. RIMS, Kyoto Univ., 17 (1981), 25-63.

[5] Paramodov, V. P., Linear Differential Operators with Constant Coefficients, Springer, Berlin-Heiderberg-New York, 1970.

[6] Pták, V., Completeness and open mapping theorem, Bull. Soc, Math. France, 86 (1958), 41-74.

[7] Treves, F., Topological Vector Spaces, Distributions and Kernels, Academic Press, New York-London, 1967. 
